\title{
Magnetic, electronic, and vibrational properties of metal and fluorinated metal phthalocyanines
}

\author{
O. I. Arillo-Flores, ${ }^{1}$ M. M. Fadlallah, ${ }^{2,3}$ C. Schuster, ${ }^{2}$ U. Eckern, ${ }^{2}$ and A. H. Romero ${ }^{1,4, *}$ \\ ${ }^{1}$ CINVESTAV-Querétaro Libramiento Norponiente No. 2000 Real de Juriquilla 76230 Querétaro, Qro, México \\ ${ }^{2}$ Institut für Physik, Universität Augsburg, 86135 Augsburg, Germany \\ ${ }^{3}$ Physics Department, Faculty of Science, Benha University, Benha, Egypt \\ ${ }^{4}$ Max-Planck-Institute für Mikrostrukturphysik, Weinberg 2, D-06120 Halle, Germany
}

(Received 15 April 2012; published 9 April 2013)

\begin{abstract}
The magnetic and electronic properties of metal phthalocyanines $(M \mathrm{Pc})$ and fluorinated metal phthalocyanines $\left(\mathrm{F}_{16} M \mathrm{Pc}\right)$ are studied by means of spin density functional theory (SDFT). Several metals $(M)$ such as Ca, all first $d$-row transition metals, and $\mathrm{Ag}$ are investigated. By considering different open shell transition metals it is possible to tune the electronic properties of $M \mathrm{Pc}$, in particular the electronic molecular gap and total magnetic moment. Besides determining the structural and electronic properties of $M \mathrm{Pc}$ and $\mathrm{F}_{16} M \mathrm{Pc}$, the vibrational modes of the ScPc-ZnPc series have been studied.
\end{abstract}

DOI: 10.1103/PhysRevB.87.165115

PACS number(s): 75.50.Xx, 31.15.ae, 71.15.Mb

\section{INTRODUCTION}

Magnetism or magnetic moments are rarely found in organic compounds, but metal phthalocyanine $(M \mathrm{Pc})$ molecules are notable exceptions. ${ }^{1}$ The $M P c$ family represents metalorganic semiconductors that display high thermal and chemical stability. ${ }^{2}$ These molecules have recently attracted large interest in applications such as in solar cells, optoelectronic devices, light-emitting diodes, thin-film transistors, and gas sensors. ${ }^{3-7}$ The magnetic properties of CoPc are used in spintronic devices. ${ }^{8}$ Furthermore, the $M P c$ are simple magnetic molecules which can serve as prototypical systems to study the magnetic properties of the $3 d$ transition metals embedded in an organic surrounding.

Fluorinated metal-phthalocyanines $\left(\mathrm{F}_{16} M \mathrm{Pc}\right)$ are also widely used in applications. In contrast to the $M P c$ which usually are $p$-type-like semiconductors, $\mathrm{F}_{16} M \mathrm{Pc}$ are used as $n$-type semiconductors in photovoltaic devices and in lightemitting diodes. Organic field-effect transistors have been intensively studied as components for low price, large area, and flexible circuit applications. To build them, an $n$-type organic semiconductor is needed. Experimental investigations focus on $\mathrm{F}_{16} \mathrm{CuPc}$ (Refs. 9 and 10) and $\mathrm{F}_{16} \mathrm{CoPc} .{ }^{1-14}$ They have been also considered as an alternative to inorganic field-effect transistors (FETs) in some specific circuit applications such as radio frequency identification cards (RFIDs), ${ }^{15,16}$ electronic paper, ${ }^{17}$ sensors, ${ }^{18,19}$ and switching devices for active matrix flat panel displays (AMFPDs). ${ }^{20}$

Several experimental techniques such as X-ray spectroscopy, ${ }^{21-25}$ neutron diffraction, ${ }^{21-25}$ scanning tunneling microscopy (STM), ${ }^{26,27}$ nuclear magnetic resonance, ${ }^{28}$ and photoemission spectroscopy ${ }^{29}$ have been used to characterize the structural and electronic properties of $M \mathrm{Pc}$. However, for further developments in the synthesis and production of novel materials and devices based on $M P c$, more accessible and common techniques such as IR and Raman spectroscopy are useful. For example, Raman scattering has already been employed to identify different metal phthalocyanines and to probe polymorphic changes (packing), ${ }^{30}$ as well as structural modifications. ${ }^{31}$ Therefore, a systematic study of vibrational modes for the series $M P c$ will provide information on the resolution of spectral features obtained with ultraviolet photoelectron, x-ray photoemission, and photoabsortion spectroscopy measurements. Vibrational coupling effects have been found to have an important effect in the description of outer molecular orbitals of $\mathrm{CuPc}^{24}$ as well as of valence bands and core levels of PbPc films. ${ }^{32}$ The study of vibrational properties can help to improve the assessment of other theoretical methods used to study metal-organic complexes, ${ }^{33}$ and to correlate magnetic transitions to changes of the surrounding. ${ }^{34}$

From a theoretical point of view, density functional theory (DFT) is a widely used method to determine the electronic structure of various organic molecules, including $M P c .{ }^{31,35,36}$ Bialek et al. applied an all-electron full-potential linearized augmented plane-wave method to study the electronic structure and magnetic moments of $\mathrm{NiPc}, \mathrm{CuPc}, \mathrm{FePc}$, and CoPc. ${ }^{37-40}$ Marom et al. have tested a variety of exchange correlation functionals for copper phthalocyanine, ${ }^{41}$ comparing local functionals such as LDA (local-density approximation) $)^{42}$ and GGA (generalized gradient approximation) ${ }^{43,44}$ with the semiempirical hybrid functional $\mathrm{B} 3 \mathrm{LYP},{ }^{45}$ the nonempirical PBE, ${ }^{46}$ PBE hybrid (PBEh), ${ }^{47}$ and the screened HSE. ${ }^{48}$ While all these functionals describe the geometrical structure quite well, the local functionals underestimate the binding of orbitals at the molecule center. Additionally, GGA $+U$ calculations have been performed in Ref. 49 to take into account the strong correlations that could be relevant around the metal center.

The present paper is organized as follows. After a brief description of the computational method in Sec. II, we report in Sec. III our results for the magnetic and electronic structure of $\mathrm{CaPc}$, of the sequence of $3 d M \mathrm{Pc}(M=\mathrm{Sc}, \mathrm{Ti}, \mathrm{V}, \mathrm{Cr}, \mathrm{Mn}$, $\mathrm{Fe}, \mathrm{Co}, \mathrm{Ni}, \mathrm{Cu}, \mathrm{Zn}$ ), and of $4 d \mathrm{AgPc}$. In addition, we compare the electronic properties of the fluorinated $M \mathrm{Pc}\left(\mathrm{F}_{16} M \mathrm{Pc}\right)$ and $M P c$. In Sec. IV we present our results for the vibrational properties of $M \mathrm{Pc}$, and we analyze general trends. A brief summary is given in Sec. V.

\section{COMPUTATIONAL METHODS}

In the present work, we focus on studying the electronic structure near the Fermi energy $E_{F}$ and the magnetic structure by means of density functional theory. Since hybrid functionals with a stronger amount of Fock exchange tend to overestimate 
magnetic moments and magnetic order, we apply the simplest exchange correlation functional, namely the LDA, as implemented in the SIESTA package. ${ }^{50}$ The electronic structure and the vibrational modes are calculated in $M \mathrm{Pc}$ and $\mathrm{F}_{16} M \mathrm{Pc}$ by using this implementation. The calculations have been performed by considering a finite spin polarization to allow for the formation of local magnetic moments. The PBE method ${ }^{46}$ is also employed in order to check the density of states (DOS) evaluated with LDA.

Wavefunctions in SIESTA are described by a local atomic orbitals basis set; we have used a double $\zeta$ basis set and an energy cutoff of $300 \mathrm{Ry}$. The pseudopotentials are norm conserving in fully nonlocal form. Structural optimizations were performed using the conjugate gradient method until the net force on every atom was smaller than $0.04 \mathrm{eV} / \AA$. Nonsymmetrical constraints were employed. At the optimized geometries force constants were evaluated and employed to calculate vibrational modes and frequencies through the dynamical matrix. As we are interested in the electronic structure and vibrational properties of single molecules, we restrict our calculations to the $\Gamma$ point, and a $28 \AA \times 27 \AA \times$ $45 \AA$ supercell was used. Further geometry optimization and frequency calculations of selected systems were also carried out with B3LYP/6-31G** in the Gaussian 03 suite of packages, in order to identify, by comparison with previous results, the IR and Raman representations.

\section{MAGNETIC AND ELECTRONIC PROPERTIES OF METAL PHTHALOCYANINE}

Metal phthalocyanine, $M \mathrm{C}_{32} \mathrm{~N}_{8} \mathrm{H}_{16}$, is an aromatic molecule with the molecular structure shown in Fig. 1. Hexadecafluorophthalocyaninatometal, $\mathrm{F}_{16} M \mathrm{Pc}$, is constructed by replacing the hydrogen atoms within $M \mathrm{Pc}$ with fluor atoms. It is expected that the metal $(M)$ is located at the center of the molecule, displaying a square planar symmetry $D_{4 h}{ }^{51}$ The structural, electronic, and magnetic properties of $M P c$ are controlled by the particular characteristics of the metal within the inner ring. Phenyl groups contribute to the stabilization of these molecular complexes and influence their

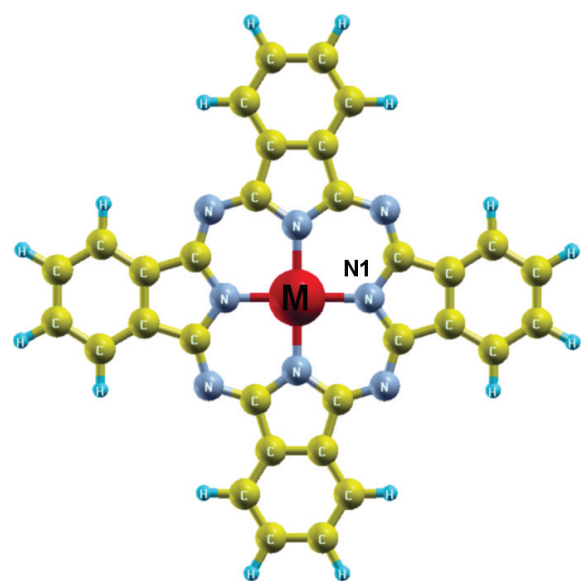

FIG. 1. (Color online) Structure under investigation: metal phthalocyanine $(M \mathrm{Pc})$ molecule. The atoms are labeled according to the chemical constituents, and the metal atom in the center is denoted by $M$.

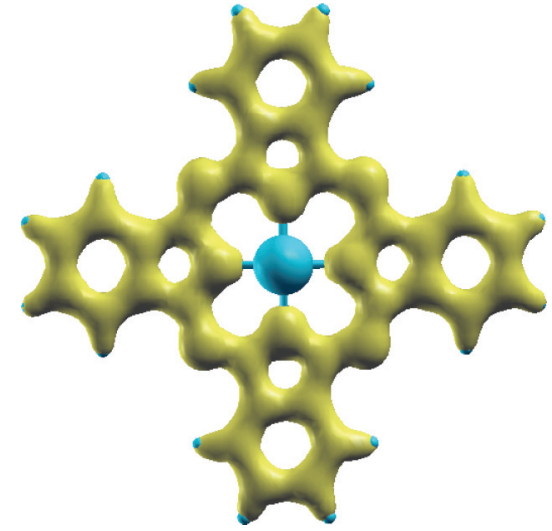

FIG. 2. (Color online) Charge density for ScPc. Isosurface value $=0.15$.

charge distribution, as reported for the formation of $\mathrm{ScPc}_{2} .{ }^{52,53}$ Nontheless, they may be subject to oxidation as in the case of TiPc (Refs. 53-55) and VPc. ${ }^{56,57}$

To study the magnetic structure of the first row transition metals and also of $\mathrm{Ca}$ and of $\mathrm{Ag}$ within an isolated $M \mathrm{Pc}$, we first focus on the determination of their geometrical structure, and on how our observations relate to our approximations and the different molecular components.

In general the optimized structures show a planar geometry except for the $\mathrm{ScPc}$ and $\mathrm{CaPc}$, where the metal is slightly out of plane while the rest of the molecule undergoes a small bending towards the opposite direction. This effect becomes larger as the atomic radius of the metal increases, the Sc atom (with radius of $1.62 \AA$ ) is displaced by $0.24 \AA$ from the molecular plane, while $\mathrm{Ca}$ (with radius of $1.97 \AA$ ) is shifted by $1.12 \AA$. To confirm the observed trend we calculate the optimized geometry for YPc obtaining an out-of-plane displacement of $0.72 \AA$. It can be seen from Fig. 2 that for ScPc the valence

TABLE I. $M$-N1 bond lengths $(\AA)$ and magnetic moments $\left(\mu_{B}\right)$ for $M \mathrm{Pc}$ and $\mathrm{F}_{16} M \mathrm{Pc}$.

\begin{tabular}{|c|c|c|c|}
\hline$M$ & $M-\mathrm{N} 1$ bond in $M \mathrm{Pc}$ & $\begin{array}{c}M-\mathrm{N} 1 \text { bond in } \\
\mathrm{F}_{16} M \mathrm{Pc}\end{array}$ & $\begin{array}{r}\text { Magnetic } \\
\text { moment }\end{array}$ \\
\hline $\mathrm{Ca}$ & 2.322 & 2.328 & 0.00 \\
\hline Sc & 1.998 & 1.992 & 0.99 \\
\hline $\mathrm{Ti}$ & 1.974 & 1.988 & 2.00 \\
\hline V & 1.968 & 1.974 & 3.00 \\
\hline $\mathrm{Cr}$ & 1.971 & 1.975 & 4.00 \\
\hline $\mathrm{Mn}$ & 2.012 & 2.021 & 4.80 \\
\hline $\mathrm{Fe}$ & $1.912,1.927^{\mathrm{a}}$ & 1.991 & 2.00 \\
\hline Co & $1.883,1.912^{\mathrm{b}}$ & 1.888 & 1.00 \\
\hline $\mathrm{Ni}$ & $1.875,1.83^{\mathrm{c}}$ & 1.884 & 0.00 \\
\hline $\mathrm{Cu}$ & $1.937,1.935^{\mathrm{d}}$ & 1.947 & 1.00 \\
\hline $\mathrm{Zn}$ & $1.975,1.980,^{\mathrm{e}} 1.954^{\mathrm{f}}$ & 1.988 & 0.00 \\
\hline $\mathrm{AgPc}$ & 2.042 & 2.044 & 0.95 \\
\hline
\end{tabular}

${ }^{\text {a Reference } 22 .}$

${ }^{\mathrm{b}}$ Reference 59 .

${ }^{\mathrm{c}}$ Reference 60.

${ }^{\mathrm{d}}$ Reference 21.

${ }^{\text {e Reference } 23 .}$

${ }^{\mathrm{f}}$ Reference 61. 

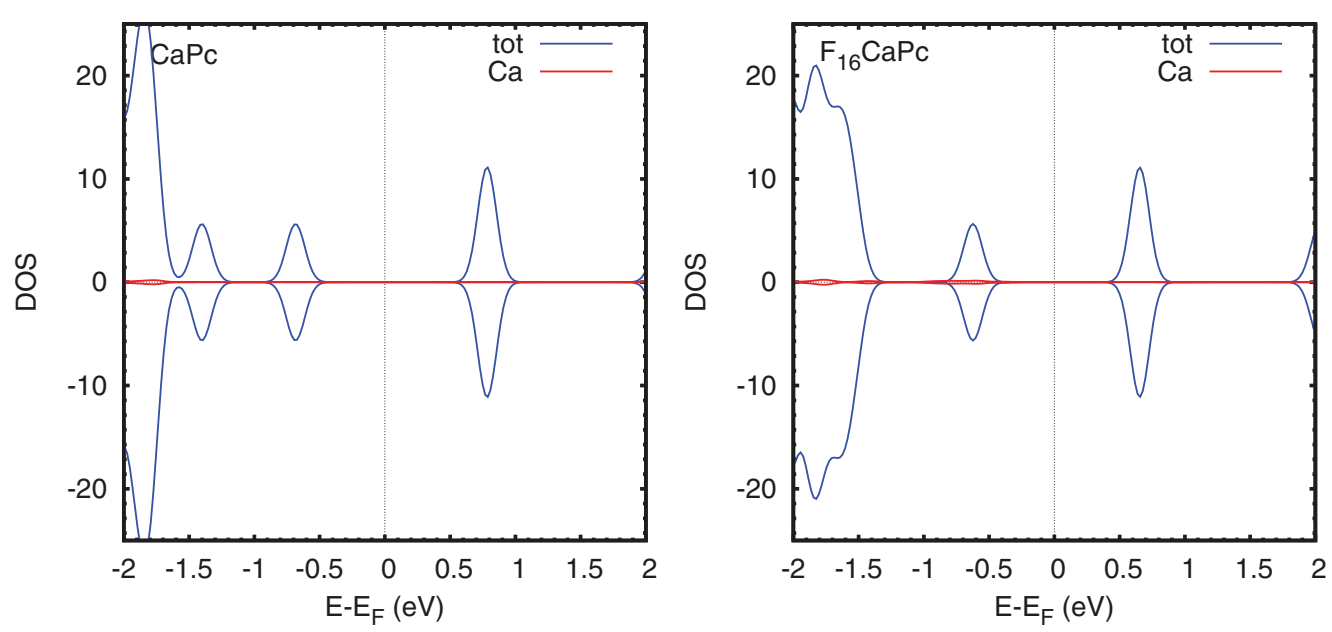

FIG. 3. (Color online) Density of states $\mathrm{CaPc}$ (left) and for $\mathrm{F}_{16} \mathrm{CaPc}$ (right).

electron density (isosurface value $=0.15$ ) appears distant from the metal center, beyond the van der Waals radium of Sc, therefore the metal departure from the molecular plane may be caused by the repulsion between inner electron shells on the metal and on adjacent atoms. In the distorted geometry the metal is more exposed and the molecular liability increases.

Geometrical structures of minimal energy may be influenced by a Jahn-Teller distortion, which tends to reduce the symmetry from $D_{4 h}$ to $D_{2 h}$. As the point-group symmetry was not constrained through our geometry optimizations, strictly speaking we have obtained, for MPc planar geometries, the $D_{2 h}$ symmetry with two $M$-N distance values slightly different from each other, nevertheless they differ only by less than $1 \%$, this variation being in the order of magnitude comparable to the precision of the energy minimization procedure. A detailed account of the Jahn-Teller distortion effect on the studied MPc series is beyond the scope of the present work. Interested readers are referred to the work of Marom and Kronik ${ }^{58}$ who emphasize the theoretical and experimental difficulties to describe precisely the electronic states of MnPc and FePc, the differences being due to their intricate potential energy surface.

Table I shows the bond lengths between the metal and the nitrogen N1, as indicated in Fig. 1. When available, we compare to experimental data. The theoretical bond lengths differ from experimental lengths by less than $0.05 \AA$, i.e., by $1-2 \%$. Decreasing the atomic covalent radius leads to a decrease in the bond length from $\mathrm{Sc}$ to $\mathrm{Cr}$, as well as from $\mathrm{Mn}$ to $\mathrm{Cu}$. In $\mathrm{F}_{16} M \mathrm{Pc}$, the $M$-N1 bond length is typically slightly larger than in $M P c$, as shown in Table I. The influence of the larger radius of fluorine with respect to hydrogen and its $p$ electrons show up only when no metallic states are present near the Fermi level.

The last row of Table I shows the magnetic moments of $M \mathrm{Pc}$ (in the case of $\mathrm{F}_{16} M \mathrm{Pc}$ molecules, the calculated magnetic moments are quite close to the related $M \mathrm{Pc}$ ). Along the sequence from $M=\mathrm{Sc}$ to $\mathrm{Mn}$, an open $d$ shell on the metal progressively becomes half filled and the MPc magnetic moments closely follow the tendency on the metal center [Sc $\left(s=1 / 2,3 d^{1}\right)$, Ti $\left(s=1,3 d^{2}\right), \mathrm{V}\left(s=3 / 2,3 d^{3}\right), \mathrm{Cr}$ $\left(s=2,3 d^{4}\right)$, and $\left.\mathrm{Mn}\left(s=5 / 2,3 d^{5}\right)\right]$. The magnetic moment found for $\mathrm{MnPc}, 5 \mu_{B}$, is larger than the previously reported value of $3 \mu_{B},{ }^{62}$ however, high magnetic moments within this series are also obtained using PBE. In the second half of the $d$ shell, total spin values lie between 0 and 2 . In general, they are smaller than those predicted for the first half. The values found for this interval agree very well with earlier results. ${ }^{2,63,64}$ Therefore, the trend in the magnetic moment can be correlated with the electron pairing and the crystal field created by the molecule to the metallic atom, which reduces the covalent character. The orbitals hybridize originating a new mixing, which alters the expected orbital ordering from plain crystalline field effects over the normal atomic orbitals. As a consequence there is an abrupt decrease of magnetic moment from $\mathrm{Mn}$ to $\mathrm{Fe}$, and zero magnetic moments are found for NiPc before completing the $d$ shell. CuPc $\left(s=1 / 2,3 d^{9}\right)$ and $\operatorname{AgPc}\left(s=1 / 2,4 d^{9}\right)$ have a hole in their nearly full $d$ shell. The fact that magnetic moments for $\mathrm{F}_{16} M \mathrm{Pc}$ are similar to those for $M P c$ suggests that molecular magnetic moments are strongly influenced by contributions to the total electronic density coming from the metal center, with less dependence on the molecular environment; this observation holds for $\mathrm{Ag}$ and even $\mathrm{Ca}$, an $s$-block element.

The observed tendency for $M-\mathrm{N} 1$ bond lengths results from two main factors. The first one is the metal atomic radius reduction along the period, which clearly dominates from $\mathrm{Ca}$ to $\mathrm{V}$, and the second one is the increase of electronic repulsion for high spin values and degree of orbital occupancy; these
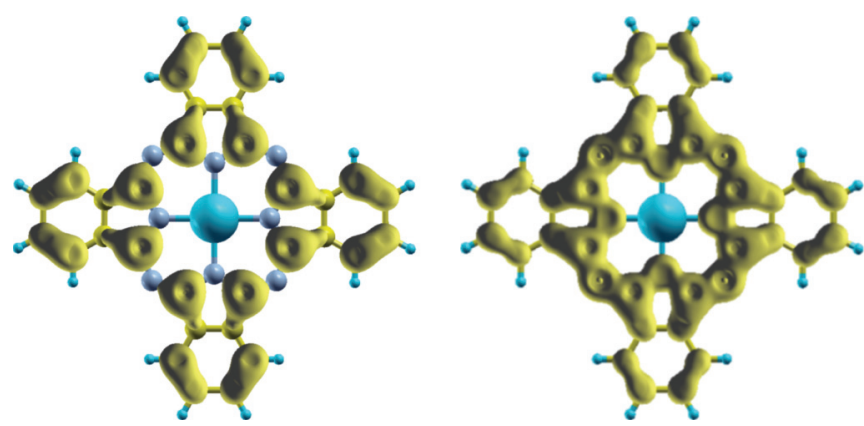

FIG. 4. (Color online) Charge density isosurface for CaPc: HOMO (left), LUMO (right). 

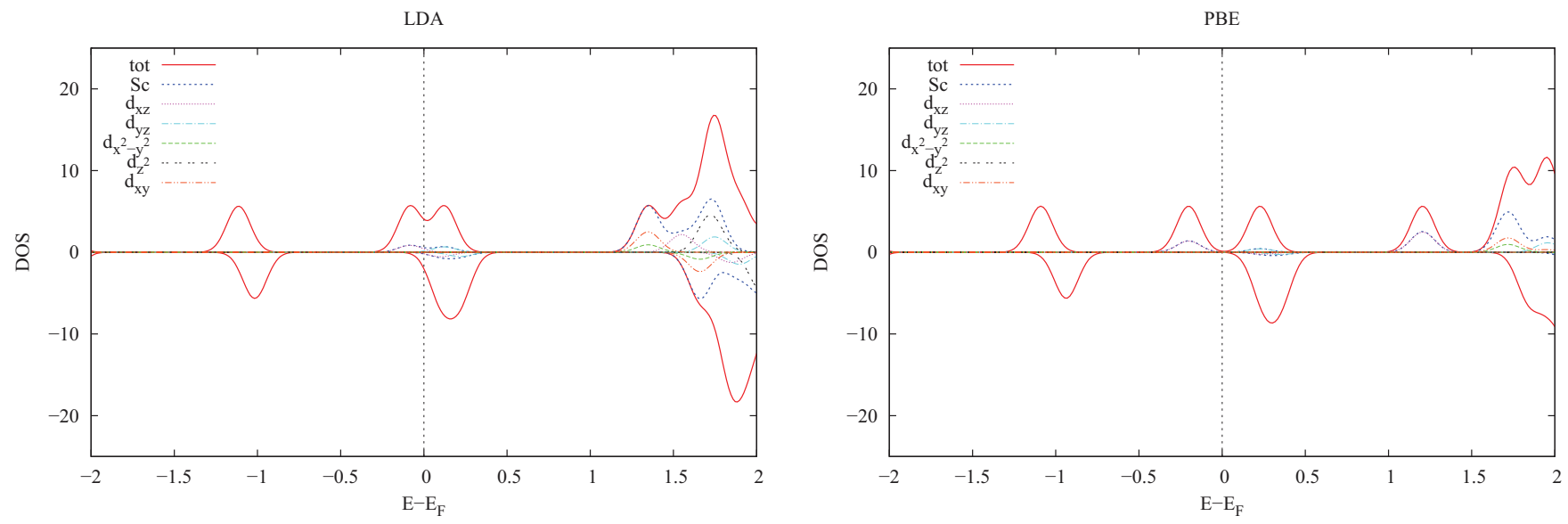

FIG. 5. (Color online) Density of states of ScPc with LDA (left) and PBE (right).

effects overcome the first one for $\mathrm{Cr}$. In the second half of the $d$ row, the decrease in magnetic-moment values is reflected in the $M-\mathrm{N} 1$ bond lengths variation from $\mathrm{Fe}$ to $\mathrm{Cu}$. Deviation from such behavior in the case of $\mathrm{ZnPc}$ may be understood by electron accumulation on the metal when the $d$ shell is full, and the increase of electronic repulsion which results.

To complement the analysis of the magnetic properties of the $M P c$, we discuss their spin resolved electronic structure in the following. First, we consider the density of states (DOS) of $\mathrm{CaPc}$ and $\mathrm{F}_{16} \mathrm{CaPc}$ shown in Fig. 3. Here and in the following plots, the density of states of one of the two spin components is multiplied by -1 . Additionally, a level broadening of molecular orbital energies with a spread of $0.1 \mathrm{eV}$ is used to simulate thermal and anharmonic effects in the spectra. ${ }^{65}$ The energy is scaled with respect to the Fermi level $E_{F}$, estimated using the Fermi-Dirac function with an electronic temperature of $300 \mathrm{~K}$. The energy gap of $\mathrm{CaPc}$, estimated as the difference between its highest occupied molecular orbital (HOMO) and its lowest unoccupied molecular orbital (LUMO), is $1.3 \mathrm{eV}$. As it can be noted in Fig. 3, these orbitals have no metal contribution, and they are localized upon the Pc atoms. Both peaks are very similar to the fluorinated derivative; the main difference is the decrease of the energy gap by $0.2 \mathrm{eV}$. However, the shape of the HOMO- 1 (first orbital with an energy below the HOMO energy) is quite different: it is very broad in $\mathrm{F}_{16} \mathrm{CaPc}$, in contrast to $\mathrm{CaPc}$ where it is rather narrow, indicating several energy states close by.

To visualize the orbital contributions to the electronic density, we calculate the energy resolved density distribution $n_{\mathrm{LDOS}}(\mathbf{r})=\int_{E_{1}}^{E_{2}} d E N(E, \mathbf{r})$, where $N(E, \mathbf{r})$ is the local density of states. The HOMO and LUMO charge density isosurfaces for $\mathrm{CaPc}$ and $\mathrm{F}_{16} \mathrm{CaPc}$ are shown in Fig. 4, in which it can be confirmed that there is no contribution from the metal atom to these orbitals. This is related to the marked propensity of the alkali metal to lose its valence electrons and to form ionic bonds, transferring the charge to the MPc backbone. Dominating contributions in the case of HOMO are from carbon atoms whereas orbitals localized on nitrogen and carbon contribute jointly to the LUMO. Therefore this state is more delocalized over the entire inner ring. The symmetry obtained for the HOMO's density indicates that it is an $a_{1 u}$ orbital while the LUMO's density concurs with an $e_{g}$ orbital type; the relative high of their corresponding DOS peak (see Fig. 3) indicates that $e_{g}$ is a doubly degenerate level.

The charge density isosurfaces for $\mathrm{F}_{16} \mathrm{CaPc}$ have almost the same form as for $\mathrm{CaPc}$. In addition, the fluor atoms have clearly visible contributions.

Turning to the $3 d$-transition metals, the DOS of ScPc and its fluorinated derivative are shown in Figs. 5 and 6. The states around $-1.1 \mathrm{eV}$ are very similar to the HOMO of CaPc. The Sc $d$ majority states show small contributions just below and above the Fermi level due to the mixing of the split $d_{x z}$ and $d_{y z}$ with the $e_{g}$ ligand orbital which, as mentioned, in $\mathrm{CaPc}$ is a LUMO doubly degenerate state. Metal contributions to unoccupied states also appear above $1.2 \mathrm{eV}$, coming from $d_{x y}$, $d_{x z}, d_{y z}$, and $d_{z^{2}}$. The PBE DOS of ScPc indicates that the $d_{x z}$ contribution to the HOMO increases while it decreases for unoccupied levels appearing at lower energies (downshift). A wider band gap of $0.3 \mathrm{eV}$ is obtained when PBE is used. Regarding the electronic structure of TiPc (Fig. 7), it can be seen that the metal has more contribution to the HOMO through its $d_{x z}$ and $d_{y z}$ orbitals than in ScPc. PBE predicts a slight HOMO downshift, a marked peak splitting of $d_{x y}$ and $d_{z^{2}}$ metallic contributions, and a band-gap increase of $0.3 \mathrm{eV}$. These effects concur with the expected reduction of the self-interaction error (SIE) for the semilocal method.

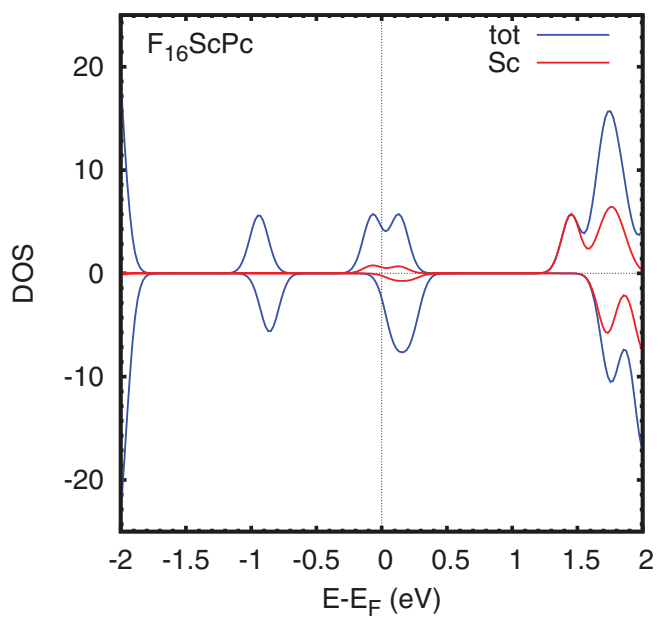

FIG. 6. (Color online) Density of states of $\mathrm{F}_{16} \mathrm{ScPc}$ with LDA. 

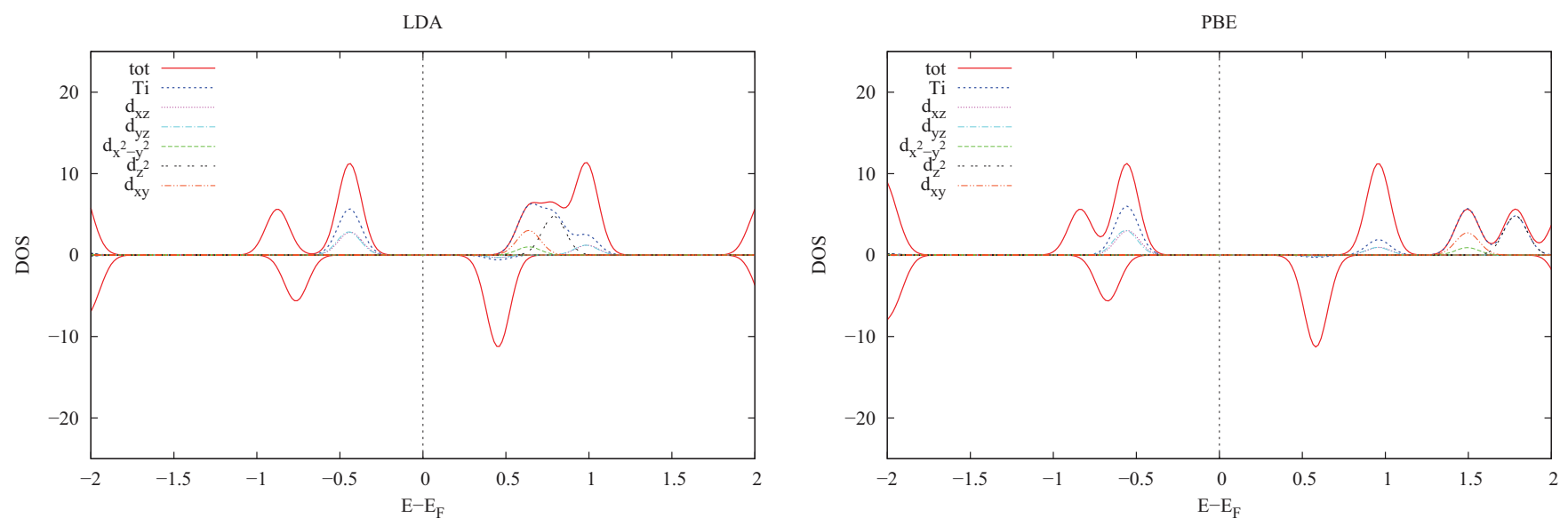

FIG. 7. (Color online) Density of states of TiPc with LDA (left) and PBE (right).

The electronic structure of $\mathrm{F}_{16} \mathrm{ScPc}$ (Fig. 6) indicates that states near the Fermi energy are not influenced by fluorination. In contrast, the main difference between TiPc and $\mathrm{F}_{16}$ TiPc (Fig. 8) is the level splitting of states with metallic contribution, particularly near the Fermi energy. The change in the admixture of $\mathrm{Ti}$ and Pc states modifies the majority DOS profile. Even though fluorination can change the electronic structure, the total magnetic moment is not altered. This is due to the charge redistribution in the molecule, as can be concluded from peak intensity changes of electronic structure states close to $E_{F}$. The charge deficiency on the metal and its magnetization, estimated as the difference between electronic charge densities of opposite spins obtained with the Mulliken population analysis, ${ }^{66}$ are reported in Table II. The table shows that, with the exception of Ti systems, the small amount of charge transferred from the metal to $\mathrm{Pc}$ is similar for the fluorinated and not fluorinated compounds. When the tendency of estimated metal magnetizations is compared to that of the total magnetizations, previously shown in Table I, it is possible to observe a close interdependence.

As noted in the second row of Table II, the estimated metal magnetization difference between TiPc and $\mathrm{F}_{16} \mathrm{TiPc}$ is $0.23 \mu_{B}$. Along with this increase there is a diminution of $0.11 \mathbf{e}$ of the

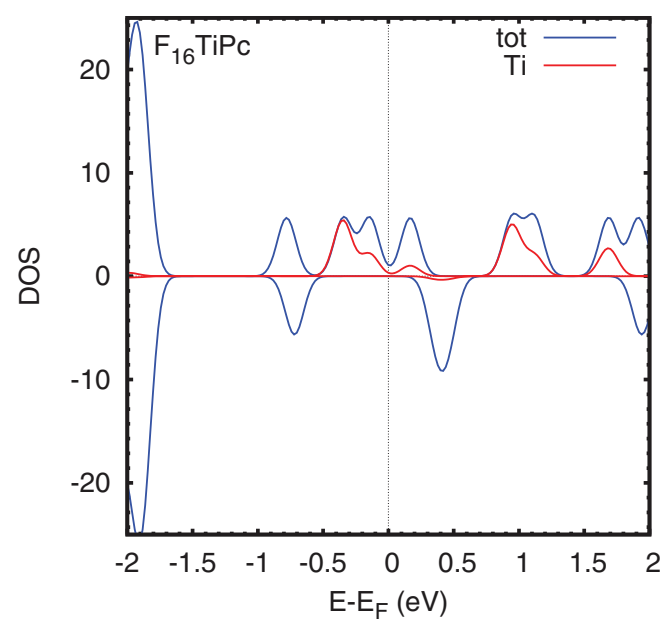

FIG. 8. (Color online) Density of states of $\mathrm{F}_{16} \mathrm{TiPc}$ with LDA. charge transferred from the metal to the rest of the molecule. This can be understood as a compensation mechanism that explains why both molecules possess equal total magnetic moments, stressing the ability of these compounds to modify their spin electronic densities by means of metal-ligand charge redistribution.

Considering the LDA majority DOS of VPc, see Fig. 9, we note that new metal orbitals contribute to the occupied states appear between the HOMO and the $a_{1 u}$ molecular orbital. They correspond to $d_{z^{2}}, d_{x y}$, and, to a lesser extent, to $d_{x^{2}-y^{2}}$. The degenerate $d_{x z}$ and $d_{y z}$ metal orbitals in the HOMO of TiPc become split in VPc, and they compose its HOMO and LUMO. The band gap is reduced to $0.4 \mathrm{eV}$. In contrast, PBE predicts this gap to be $0.9 \mathrm{eV}$, displaying a downshifted $\mathrm{HOMO}$ (upshifted LUMO) with a larger (smaller) metal contribution. Peak hights of $d_{z^{2}}$ and $d_{x y}$ are similar to the $d_{y z}$ peak at HOMO, hence they are relevant for electron filling along the $d$-row series. Their energy ordering, and particularly the position of $d_{x y}$, indicates that the crystalline field effect is not determinant. In the PBE description the $d_{z^{2}}$ and $d_{x y}$ metal orbitals appear at lower energies of $a_{1 u}$.

Following along the $3 d$ sequence, the additional electron of $\mathrm{Cr}$ causes important changes in the electronic DOS; see Fig. 10. Metal contributions to occupied levels appear below HOMO. The $d_{x z}$ and $d_{y z}$ orbitals which are split in VPc become degenerate as in TiPc. For the first time within this MPc series the closest orbitals to the Fermi energy show larger energy differences for the majority states than for the minority states. It can be seen that there are not metal contributions to HOMO and LUMO, they principally localize upon the inner ring. HOMO1 and HOMO-2 (first and second orbital below the HOMO energy) show only a small downshift with PBE but the band gap remains almost the same.

The density of states of $\mathrm{MnPc}$ and $\mathrm{FePc}$ are shown in Figs. 11 and 12, respectively. Inside the $-2.0 \ldots 2.0 \mathrm{eV}$ window around the Fermi energy, the only Mn non-negligible contribution corresponds to the $d_{x^{2}-y^{2}}\left(b_{1 g}\right)$ at HOMO. Other metal contributions to occupied states, previously seen in VPc and $\mathrm{CrPc}$, appear at energies lower than $0.5 \mathrm{eV}$ from the HOMO-1 $\left(\mathrm{a}_{1 u}\right)$. The LUMO displays only the ligand contribution, then the closest orbitals to the Fermi energy have a metal-ligand character with a narrow band gap of 
TABLE II. Band gap, metal magnetization, and metal charge densities of $M P c$ and $\mathrm{F}_{16}$.

\begin{tabular}{|c|c|c|c|c|c|c|}
\hline \multirow[b]{2}{*}{$M$} & \multicolumn{2}{|c|}{ Band gap (eV) } & \multicolumn{2}{|c|}{ Magnetization $\left(\mu_{B}\right)$} & \multicolumn{2}{|c|}{ Charge density (a.u.) } \\
\hline & $M \mathrm{Pc}$ & $\mathrm{F}_{16} M \mathrm{Pc}$ & $M \mathrm{Pc}$ & $\mathrm{F}_{16} M \mathrm{Pc}$ & $M \mathrm{Pc}$ & $\mathrm{F}_{16} M \mathrm{Pc}$ \\
\hline $\mathrm{Ca}$ & 1.5 & 1.3 & 0.00 & 0.00 & 1.26 & 1.27 \\
\hline $\mathrm{Sc}$ & 0.2 & 0.2 & 0.19 & 0.20 & 0.30 & 0.28 \\
\hline $\mathrm{Ti}$ & 0.9 & 0.4 & 1.47 & 1.70 & 0.28 & 0.17 \\
\hline V & 0.4 & 0.5 & 2.97 & 2.98 & 0.15 & 0.16 \\
\hline $\mathrm{Cr}$ & 1.3 & 1.2 & 4.02 & 4.03 & 0.05 & 0.07 \\
\hline $\mathrm{Mn}$ & 0.14 & 0.14 & 4.78 & 4.83 & 0.48 & 0.48 \\
\hline $\mathrm{Fe}$ & 0.4 & 0.4 & 2.58 & 2.57 & 0.30 & 0.31 \\
\hline Co & 1.2 & 1.3 & 1.31 & 1.30 & 0.23 & 0.24 \\
\hline $\mathrm{Ni}$ & 1.5 & 1.4 & 0.00 & 0.00 & 0.14 & 0.15 \\
\hline $\mathrm{Cu}$ & 1.1 & 1.4 & 0.47 & 0.47 & 0.15 & 0.16 \\
\hline $\mathrm{Zn}$ & 1.5 & 1.3 & 0.00 & 0.00 & 0.39 & 0.39 \\
\hline $\mathrm{Ag}$ & 0.4 & 0.4 & 0.38 & 0.38 & 0.25 & 0.26 \\
\hline
\end{tabular}
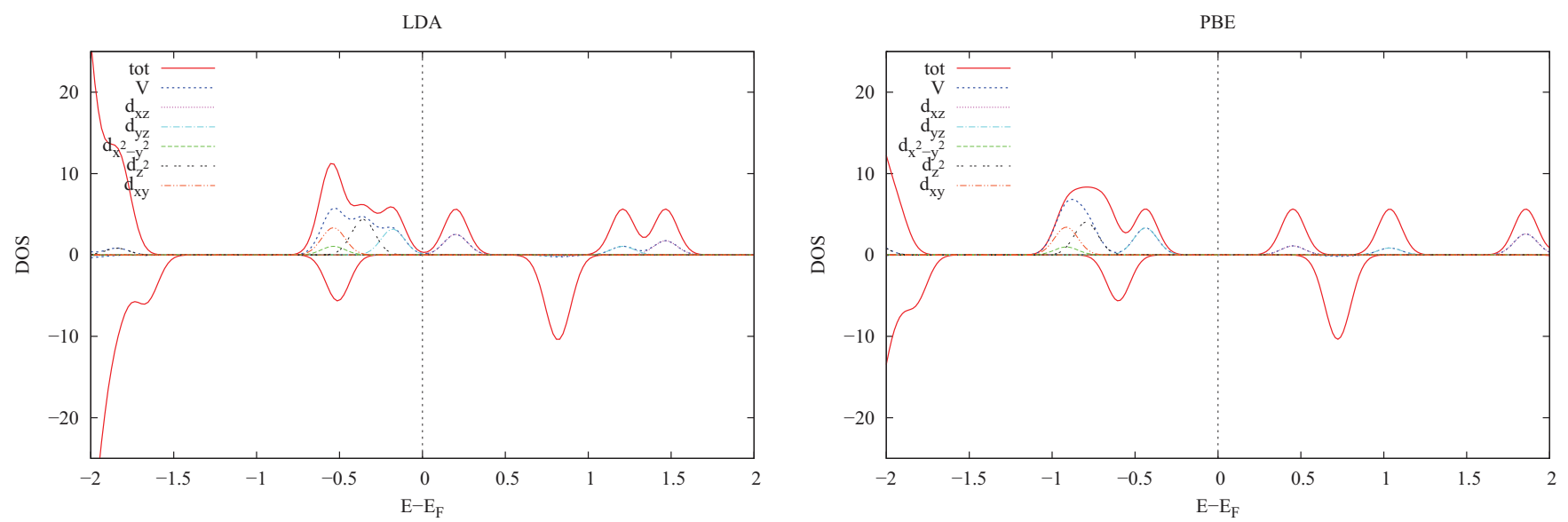

FIG. 9. (Color online) Density of states of VPc with LDA (left) and PBE (right).
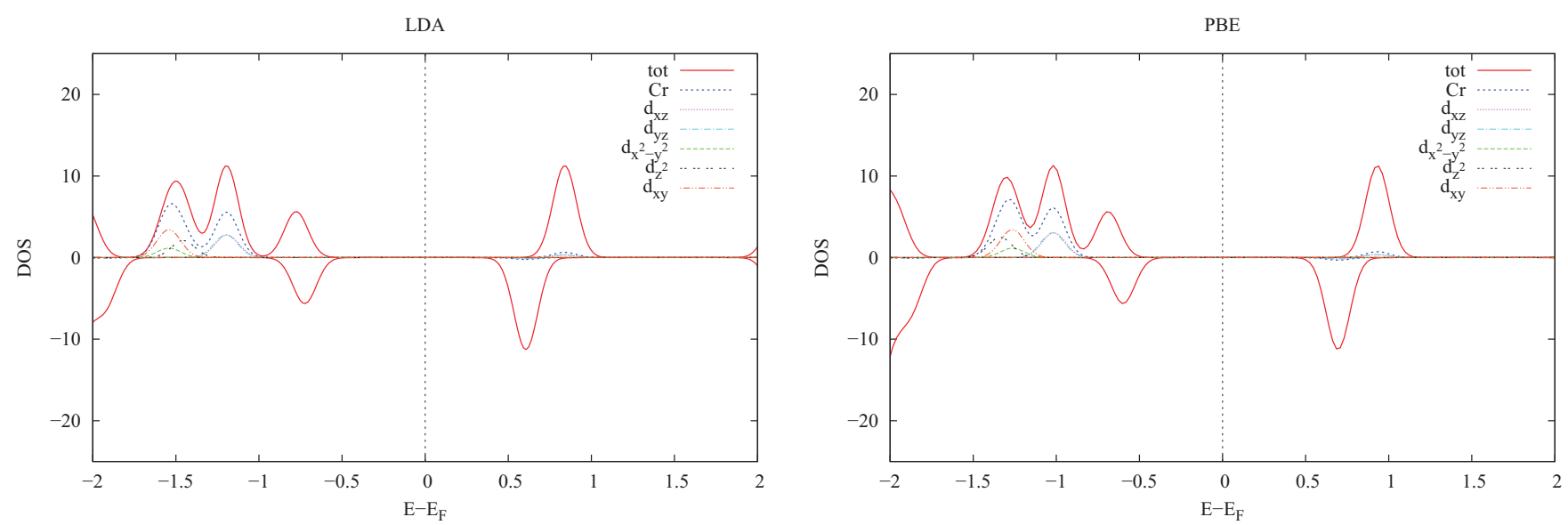

FIG. 10. (Color online) Density of states of CrPc with LDA (left) and PBE (right). 

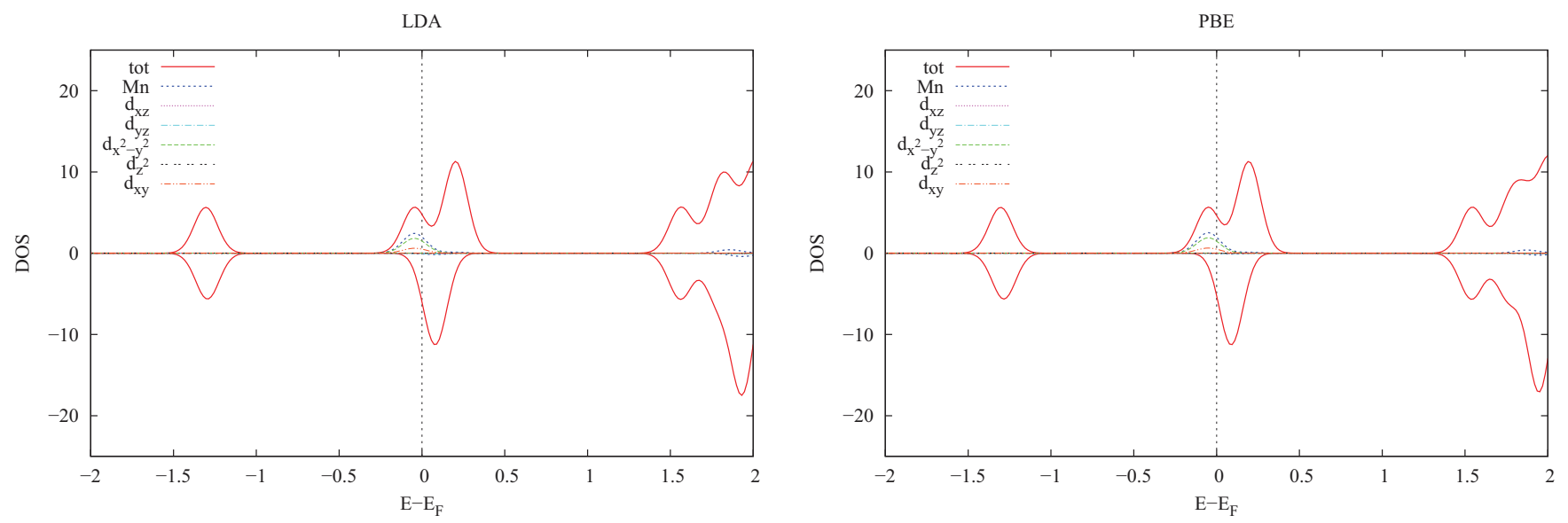

FIG. 11. (Color online) Density of states of MnPc with LDA (left) and PBE (right).

$0.14 \mathrm{eV}$ (LDA), $0.18 \mathrm{eV}$ (PBE). These values are close to the PBE result, $0.21 \mathrm{eV}$, of Stradi et al ${ }^{67}$ who used different double- $\zeta[6-31 \mathrm{G}(d, p)]$ and triple- $\zeta$ basis sets for localized Gaussian-type orbitals (GTOs), as well as plane-wave basis sets. Their peak decomposition analysis of DOS indicates that the metal contributes to HOMO and LUMO through $d_{y z}$ and $d_{x z}$; this result holds for every method they tested although it significantly reduces for LUMO with PBE0 and HSE06. Different functionals (PBE, B3LYP, PBEh, and M06) have predicted either of two configurations if symmetry is enforced, $a_{1 g} \downarrow$ (HOMO) $e_{g} \downarrow$ (LUMO) and $b_{2 g} \downarrow$ (HOMO) $e_{g} \downarrow$ (LUMO). ${ }^{58}$ On the other hand, when symmetry restrictions are not used either of two configurations are obtained: $e_{g} \downarrow(\mathrm{HOMO}) e_{g} \uparrow(\mathrm{LUMO})$ and $e_{g} \downarrow(\mathrm{HOMO}) e_{g} \downarrow(\mathrm{LUMO})$, as reported in the same work. Qualitatively, our result agrees with those configurations with a metal (ligand) contribution to the HOMO (LUMO) state. Both theoretical works point out that the calculated eigenvalue spectra of isolated MnPc appears compressed (in a smaller energy window) by about $20 \%$ with respect to the reported data of the UPS experiment for thin and thick films deposited on metal surfaces. A more direct comparison is not possible because data for the gas phase are

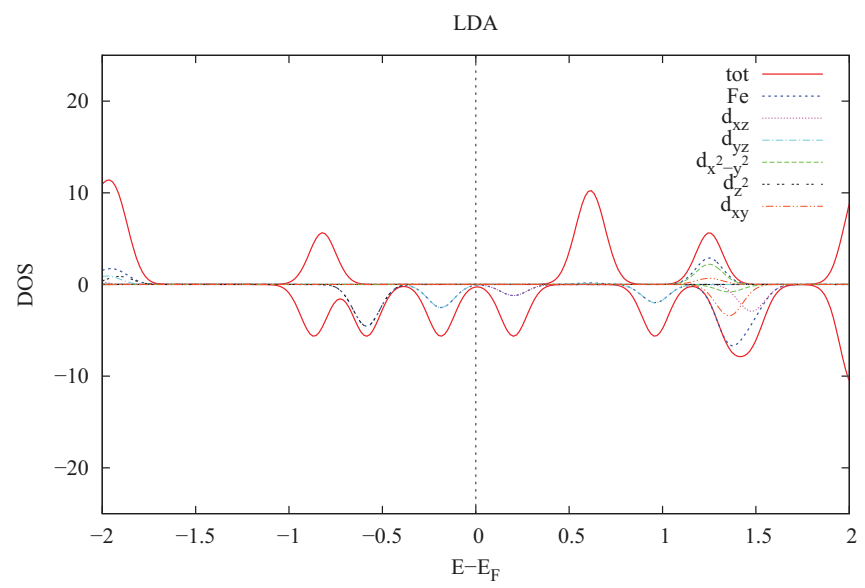

not available, and our calculations are basically for isolated molecules.

In $\mathrm{FePc}$, the addition of one electron initiates the second half of the $d$ shell, where now the atomic orbitals can have two electrons, bringing about more possibilities to accommodate electrons and increasing the electron correlation due to the electron pairing. The HOMO and LUMO can be identified as $e_{g}$ orbitals with $d_{y z}$ and $d_{x z}$ metal contributions, respectively, when using LDA, and $d_{x^{2}-y^{2}}$ metal orbital and $e_{g}$ with PBE. Regarding the metal (ligand) character of HOMO (LUMO), our PBE and LDA result concurs with recent calculations ${ }^{63,68-70}$ but deviates from previous predictions. ${ }^{58}$ Furthermore, several theoretical works devoted to the interpretation of experimental results differ in the assessment of metal or ligand character of HOMO and LUMO. The analysis of electron energy-loss spectral data for FePc films ${ }^{71}$ strongly suggests that partially occupied states $e_{g}$, with $d_{y z}$ and $d_{y z}$ metal contributions, lie in between $a_{1 u}$ and $e_{g}$ ligand states, manifesting metal participation for $\mathrm{HOMO}$ with an energy difference to LUMO ( $e_{g}$ orbital on the ligand) of $0.45 \mathrm{eV}$, very close to our LDA band gap, $0.4 \mathrm{eV}$, and PBE, $0.5 \mathrm{eV}$, estimates. The occurrence of metal orbitals close to the Fermi level, particularly for HOMO, has also been concluded

FIG. 12. (Color online) Density of states of FePc with LDA (left) and PBE (right). 

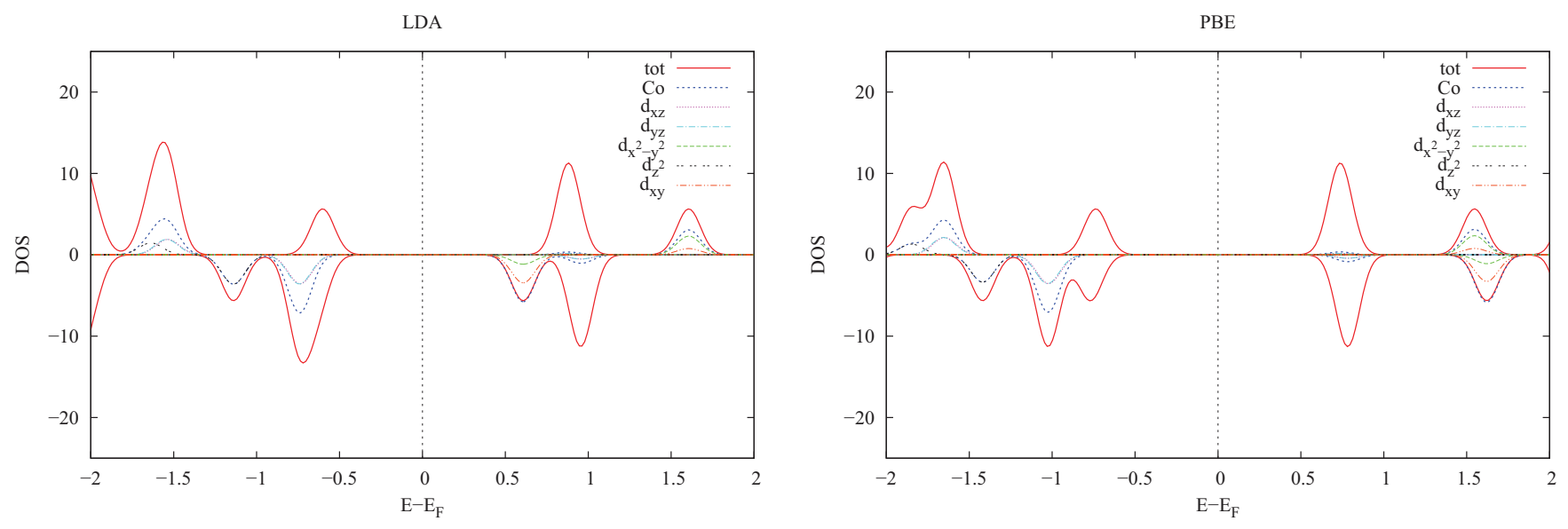

FIG. 13. (Color online) Density of states of CoPc with LDA (left) and PBE (right).

from the analysis and interpretation of photoemission and $\mathrm{x}$-ray-absorption spectroscopy measurements of $\mathrm{FePc}$ on $\mathrm{Ag}(111) .^{72}$ The metal-ligand character obtained with LDA for HOMO-LUMO is used to describe the charge transfer between $\mathrm{FePc}$ and $\mathrm{Au}(111),{ }^{73}$ by means of the $\mathrm{LDA}+U$ method. Analogously the GGA $+U$ method is employed to reproduce charge reorganization of FePc adsorbed on $\mathrm{Ag}(100)$ deduced from STM spectrospcopy. ${ }^{70}$ Apart from the $+U$ correction our results differ in a qualitative manner because we find $d_{z^{2}}$ states close the Fermi level but not $d_{x y}$; instead we get $d_{x^{2}-y^{2}}$. This may be due to a larger degree of metal-ligand hybridization for $d_{x y}$. In contrast a ligandligand character is suggested to account for photoelectron spectroscopy experiments which help to determine $U_{\text {eff }}$ within the GGA $+U$ correction. ${ }^{68}$ Nevertheless, recent total-energy density functional calculations together with density-matrix constraints indicate that isolated and columnar stacking FePc molecule $(\alpha-\mathrm{FePc})$ ground states have a substantial $d$ metal orbital contribution to HOMO. ${ }^{69}$

Figure 13 displays the density of states for CoPc. The LDA band gap, $1.2 \mathrm{eV}$, is larger than the previously reported result, $1.0 \mathrm{eV},{ }^{8}$ using LDA and GGA + vdW. The band gap obtained with GGA, $1.45 \mathrm{eV}$, is the same as the one predicted for a CoPc monolayer ${ }^{40}$ by the full-potential linearized augmented plane-wave (FLAPW) method, and very close to the GGA $+U$ result of $1.4 \mathrm{eV},{ }^{70}$ but smaller than the calculated value of $1.96 \mathrm{eV}^{35}$ using the Vosko-Wilk-Nusair (VWN) potential ${ }^{42}$ including exchange and correlation gradient corrections. In the majority component, LDA and PBE indicate that HOMO and LUMO are distributed over the Pc atoms. From the charge density isosurface (Fig. 14) of the occupied Co states from $-2 \mathrm{eV}$ to $E_{F}$, we see that the contribution to the majority density principally derives from $\mathrm{Co}$ and $\mathrm{N}$ states while carbon atoms contribute to minority states. In regard to the LDA DOS, we find that the metal-localized occupied $d_{z^{2}}$ orbital downshifts in the GGA description; on the contrary the LUMO, $d_{x y}$, upshifts and the $2 e_{g}$ orbital becomes LUMO. The ligand $\left(a_{1 g}\right)$-ligand $\left(2 e_{g}\right)$ character of HOMO-LUMO differs from the metal $\left(d_{z^{2}}\right)$-ligand, ${ }^{40}$ ligand-metal, ${ }^{64}$ ligand $\left(1 e_{g}\right)$-ligand $\left(2 e_{g}\right)$ (Ref. 35) character of other works, but agrees with recent theoretical results ${ }^{70}$ in the gas phase, carried out to account for STM experimental observations.
From the NiPc DOS functions shown in Fig. 15, it can be noted that there are no majority nor minority spin densities. The electron pairing that occurs in this close-shell system causes equal positions and the same orbital order for both spins. Metal orbitals forming occupied orbitals are found below HOMO, while at the same time there are only very small $\mathrm{Ni} d_{x z, y z}$ contributions to the LUMO orbitals giving a ligand-ligand character to HOMO-LUMO with the same band gap of $1.5 \mathrm{eV}$ for both spins. This value is smaller than the one obtained with the Hartree-Fock (HF) method, $2.41 \mathrm{eV},{ }^{38}$ but larger than the LDA prediction, $0.7 \mathrm{eV}$, for the monolayer and crystal monoclinic phase in the same work. Our band gap is very close to the estimation with the VWN potential of $1.47 \mathrm{eV},{ }^{35}$ but that study also reported that the LUMO corresponds to a $b_{1 g}$ orbital, like a later PBE evaluation. ${ }^{2}$ Regarding the band-gap value and LUMO + 1's identity, our result agrees with more recent GGA $+U$ (Ref. 70) and spectroscopy-oriented configuration interaction (SORCI) calculations. ${ }^{63}$

The DOS functions of CuPc (Fig. 16) indicates that splitting between $b_{1 g} \uparrow$ and $b_{1 g} \downarrow$ increases when the PBE functional is used, making the $a_{1 u}$ orbital the HOMO and leaving the $b_{1 g} \downarrow$ as LUMO. This up- and downshift of occupied and unoccupied orbitals, respectively, is consistent with the expected reduction

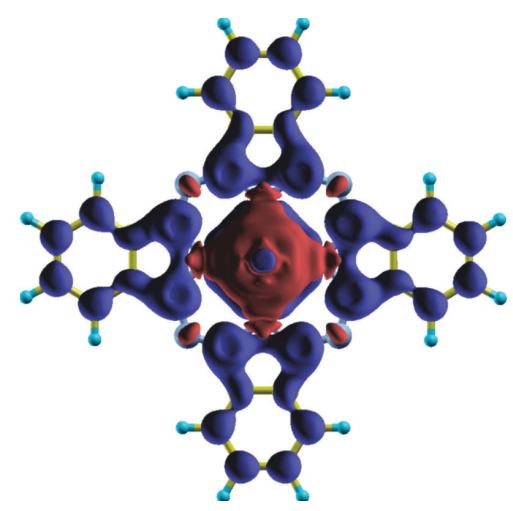

FIG. 14. (Color online) Charge density isosurface for occupied states of $\mathrm{CoPc}$, red minority-spin and blue majority-spin electron density. 

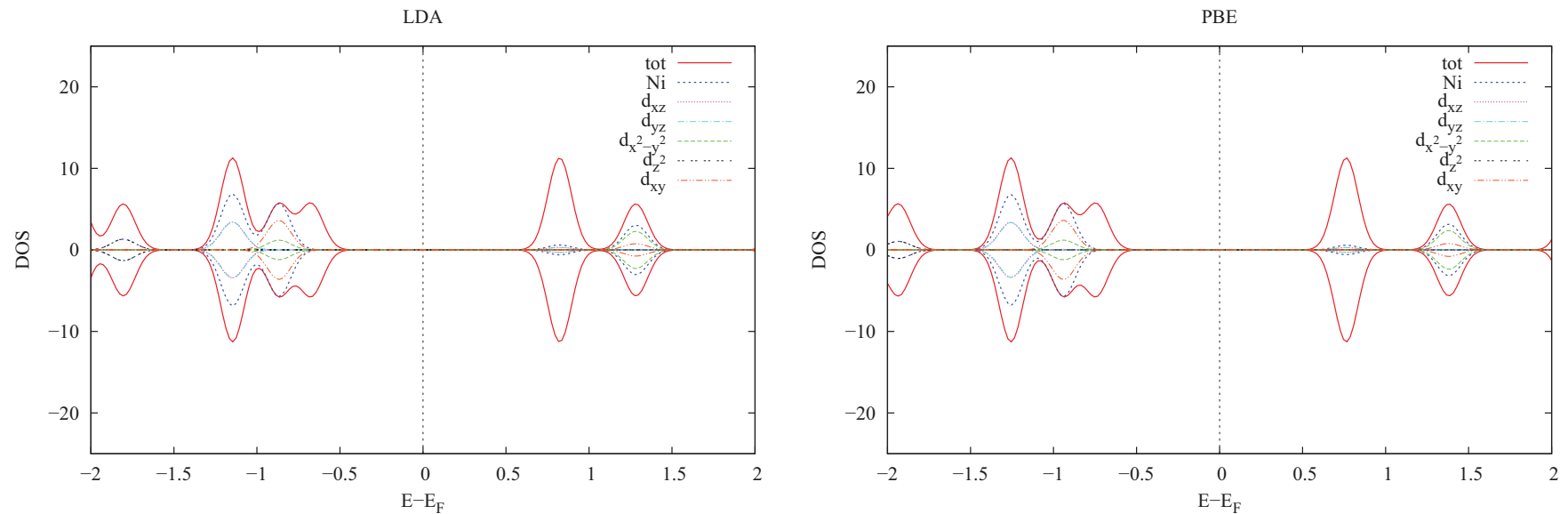

FIG. 15. (Color online) Density of states of NiPc with LDA (left) and PBE (right).
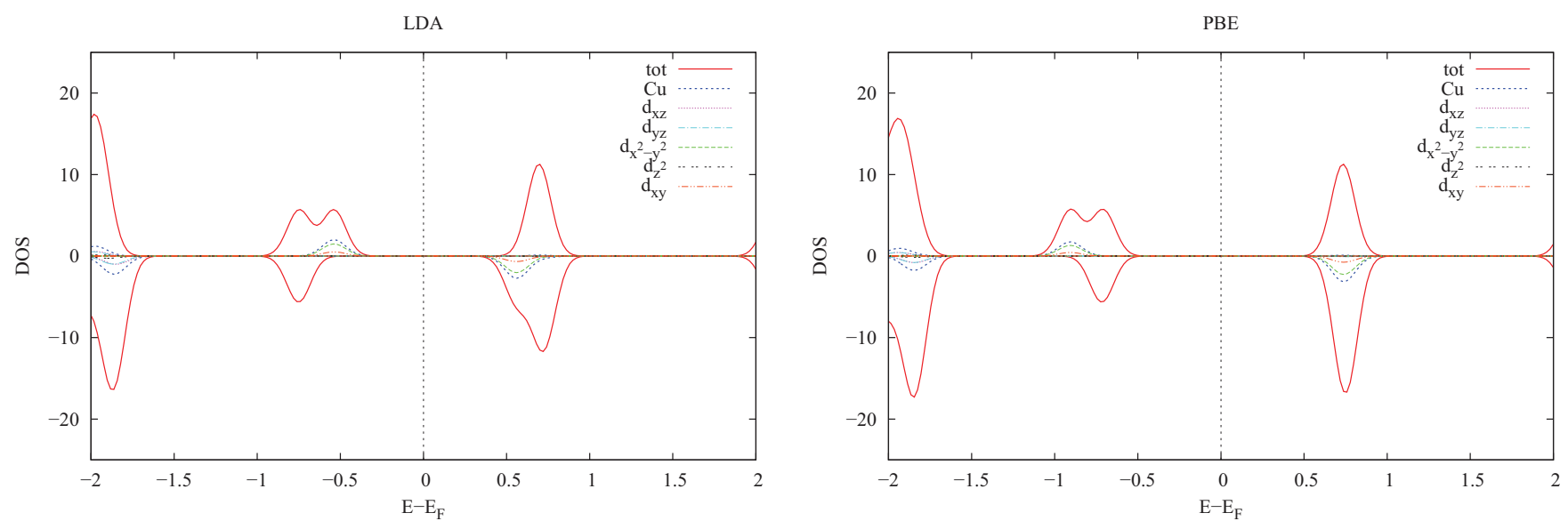

FIG. 16. (Color online) Density of states of CuPc with LDA (left) and PBE (right).
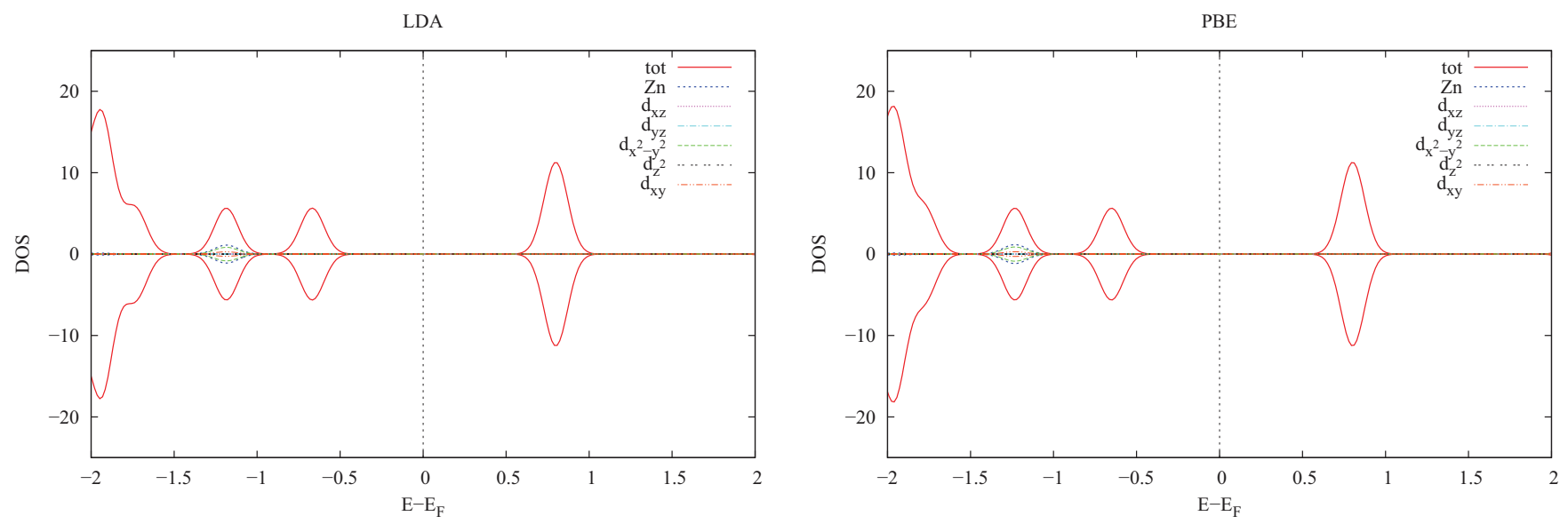

FIG. 17. (Color online) Density of states of $\mathrm{ZnPc}$ with LDA (left) and PBE (right). 
TABLE III. Frequencies $\left(\mathrm{cm}^{-1}\right)$ of relevant vibrational modes of $\mathrm{ZnPc}$, and comparison with $\mathrm{CuPc}$

\begin{tabular}{cccccc}
\hline \hline & \multicolumn{3}{c}{ ZnPc } & & CuPc \\
\cline { 2 - 3 } \cline { 5 - 5 } & Experimental $^{\mathrm{a}}$ & Calculated $^{\mathrm{a}}$ & Calculated $^{\mathrm{b}}$ & & Calculated $^{\mathrm{c}}$ \\
\hline Raman & 1505 & 1517 & 1566 & 1609 \\
& 1447 & 1438 & $1448^{\mathrm{d}}$ & & 1491 \\
& 1340 & 1362 & $1347^{\mathrm{d}}$ & & 1395 \\
& 1144 & 1004 & & 1351 \\
& & 1134 & & 1169 \\
& & 1278 & & 1035 \\
IR & 747 & 735 & 737 & 763 \\
& 727 & 703 & 706 & 730 \\
& 752 & 741 & & 769 \\
& 780 & 760 & & 786 \\
\hline \hline
\end{tabular}

${ }^{\mathrm{a}}$ From Ref. 30.

${ }^{\mathrm{b}}$ Our CA/DZP calculation.

${ }^{\mathrm{c}}$ Our B3LYP/6-31G** calculation.

${ }^{\mathrm{d}}$ Sorted only by numerical value. See text for explanation.

of the SIE effect for localized levels. The ordering agrees with recent GGA $+U$ calculation including the $1.4-\mathrm{eV}$ band gap, ${ }^{70}$ and differs from SORCI results ${ }^{63}$ and previous VWN predictions. ${ }^{35}$

The last two structures we discuss are $\mathrm{ZnPc}$ and AgPc. For $\mathrm{ZnPc}$, the $\mathrm{Zn}$ metal states overlap with $\mathrm{C}$ and $\mathrm{N}$ states at $1.2 \mathrm{eV}$ for the majority and minority states; see Fig. 17. The $\mathrm{C}$ and $\mathrm{N}$ contributions to the states are observed above $-1.2 \mathrm{eV}$, specifically at $-0.75 \mathrm{eV}$ for $\mathrm{C}$ orbitals and at $0.75 \mathrm{eV}$ for $\mathrm{C}$ and $\mathrm{N}$ orbitals. The band gap is $1.5 \mathrm{eV}$, similar to the $\mathrm{CaPc}$ molecule, but smaller than a previously reported value. ${ }^{31}$ For $\mathrm{AgPc}$, the Ag states mix with the $\mathrm{N}$ and $\mathrm{C}$ states at $-0.1 \mathrm{eV}$ for the majority states, and at $0.7 \mathrm{eV}$ for the minority states. The pure $\mathrm{C}$ states prevail at $-1.3 \mathrm{eV}, \mathrm{C}$ and $\mathrm{N}$ states at $0.2 \mathrm{eV}$ and above $1.4 \mathrm{eV}$, thus it is difficult to decide whether $\mathrm{AgPc}$ becomes metallic or semiconducting; see Fig. 18.

The band gaps for $\mathrm{F}_{16} M \mathrm{Pc}$ and for $M \mathrm{Pc}$ are given in Table II. The gap of each $\mathrm{F}_{16} M \mathrm{Pc}$ is less than or equal to the gap of the corresponding $M \mathrm{Pc}$, which can be correlated to the fact that fluor is more electronegative thus the electronic density in the central region of the molecule is more strongly attracted to the surrounding shell.

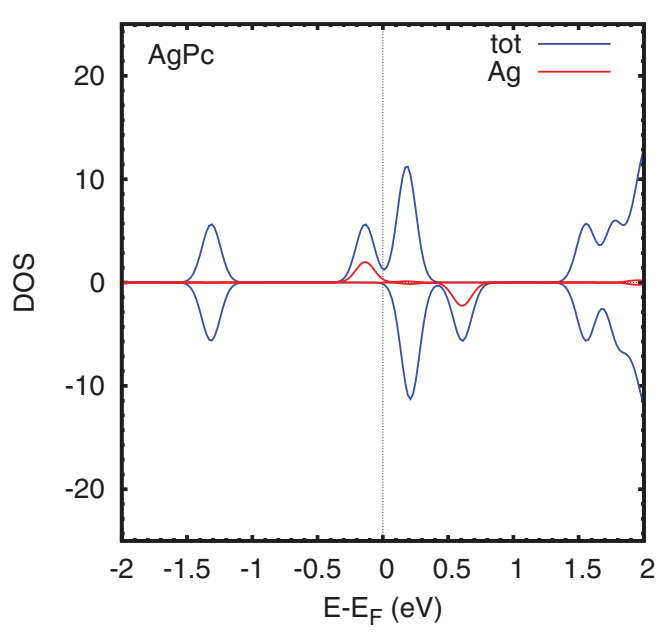

FIG. 18. (Color online) DOS of AgPc.

\section{VIBRATIONAL PROPERTIES OF METAL PHTHALOCYANINES}

The comparison of the calculated frequencies with experimental data of $\mathrm{ZnPc}$ indicates a semiquantitative agreement for the main characteristics of Raman and infrared (IR) spectra (Table III). The experimental Raman spectra presented in Fig. 7 from the work of Tackley et al. ${ }^{30}$ display three bands which are more intense than the rest; our results coincide with their positions within $10 \mathrm{~cm}^{-1}$ with the exception of the most intense band at $1505 \mathrm{~cm}^{-1}$.

Our calculated frequencies of 1448 and $1347 \mathrm{~cm}^{-1}$ are close to the experimental bands at 1447 and $1340 \mathrm{~cm}^{-1}$, however, their displacement eigenvectors have more similarities with those found in previously reported vibrational modes at 1438 and $1278 \mathrm{~cm}^{-1}$, respectively (see Supplemental Information of Ref. 30). The IR and Raman nature of each vibrational mode considered here is compared to and confirmed through the displacement vector analysis of the calculated results for CuPc, also shown in Table III. The match between each vibrational mode type (IR) between $\mathrm{ZnPc}$ and $\mathrm{CuPc}$ gives us the confidence to perform the same comparative analysis with the rest of the $M P c$ studied here.

Tables IV and V show calculated frequencies of the $M \mathrm{Pc}$ complexes for relevant vibrational modes such as breathing, and for those which involve atomic displacement near the metal. Displacement vectors for such modes are schematically presented in Figs. 19 and 20. As expected, symmetric

TABLE IV. Frequencies $\left(\mathrm{cm}^{-1}\right)$ of relevant vibrational modes for $M P c$.

\begin{tabular}{|c|c|c|c|c|c|c|c|c|c|c|}
\hline Mode description & Sc & $\mathrm{Ti}$ & $\mathrm{V}$ & $\mathrm{Cr}$ & Mn & $\mathrm{Fe}$ & Co & $\mathrm{Ni}$ & $\mathrm{Cu}$ & $\mathrm{Zn}$ \\
\hline Metal out of plane & 85 & 122 & 108 & 144 & 54 & 110 & 168 & 184 & 148 & 110 \\
\hline Asymm. breathing & & 193 & 205 & 201 & 171 & 175 & 191 & 197 & 174 & 159 \\
\hline Symm. breathing & & & 262 & 262 & 229 & & 260 & 255 & 259 & 264 \\
\hline $\mathrm{Ip}^{\mathrm{a}} \mathrm{N}-M-\mathrm{N}$ bending & 194 & 203 & 209 & 210 & 202 & 208 & 243 & 243 & 220 & 218 \\
\hline $\mathrm{Op}{ }^{\mathrm{b}}$ asymm. $M-\mathrm{N}_{4}$ bending & & 279 & & 275 & & 243 & 314 & & 250 & 223 \\
\hline $\mathrm{Op}^{\mathrm{b}}$ symm. $M-\mathrm{N}_{4}$ bending & 342 & 351 & 381 & 345 & & 361 & 372 & 394 & 331 & 226 \\
\hline
\end{tabular}

\footnotetext{
${ }^{\mathrm{a}} \mathrm{Ip}=$ in plane.

${ }^{\mathrm{b}} \mathrm{Op}=$ out of plane.
} 
TABLE V. Frequencies $\left(\mathrm{cm}^{-1}\right)$ of relevant vibrational modes for $M$ Pc.

\begin{tabular}{|c|c|c|c|c|c|c|c|c|c|c|}
\hline Mode description & $\mathrm{Sc}$ & $\mathrm{Ti}$ & $\mathrm{V}$ & $\mathrm{Cr}$ & $\mathrm{Mn}$ & $\mathrm{Fe}$ & $\mathrm{Co}$ & $\mathrm{Ni}$ & $\mathrm{Cu}$ & $\mathrm{Zn}$ \\
\hline$M-\mathrm{N}_{2}$ stretching-bending & 326 & 341 & 352 & 357 & 312 & 309 & 389 & 345 & 301 & 241 \\
\hline$M-\mathrm{N}_{2}$ stretching-bending & 329 & 342 & 358 & 363 & 316 & 363 & 401 & 351 & 302 & 245 \\
\hline$M-\mathrm{N}_{4}$ asymm. stretching & 544 & 545 & 542 & 546 & 537 & 511 & 546 & 542 & 539 & 533 \\
\hline$M-\mathrm{N}_{4}$ symm. stretching & 576 & 574 & 572 & 576 & 569 & 575 & 581 & 578 & 577 & 576 \\
\hline$M-\mathrm{N}_{4}$ asymm. stretching & 1325 & 1183 & 1205 & 1357 & 1303 & 1342 & 1359 & 1362 & 1351 & 1347 \\
\hline$M-\mathrm{N}_{4}$ symm. stretching & 1366 & 1337 & 1387 & 1401 & 1348 & 1375 & 1407 & 1407 & 1403 & 1409 \\
\hline
\end{tabular}

vibrational modes are found at higher frequencies than asymmetric modes, but their frequency shift, when the metal center is varied, depends on the particular mode. Symmetric and asymmetric breathing movements of the entire molecule show similar magnitudes of frequency shifts, the main difference being that frequencies of asymmetric breathing modes increase when going from $\mathrm{Zn}$ to $\mathrm{Co}$, while the opposite tendency is found for the symmetric modes (see Fig. 21). This behavior may be explained by the release of stress around the metal inside the ring when it opens simultaneously in all directions.

In the case of the $M-\mathrm{N}_{4}$ stretching modes, it is apparent from Fig. 21 that there is a consistent increase from $\mathrm{Zn}$ to $\mathrm{Co}$ or $\mathrm{Ni}$ for the asymmetric type. The high-frequency $\left(>1180 \mathrm{~cm}^{-1}\right)$ modes of $M-\mathrm{N}_{4}$ stretching have larger shifts. Their frequencies decrease from $\mathrm{Fe}$ to $\mathrm{Co}$, then they increases again for $\mathrm{Cr}$. These variations are not present for low frequency $\left(<600 \mathrm{~cm}^{-1}\right)$

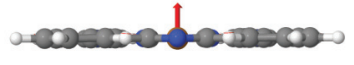

(a)

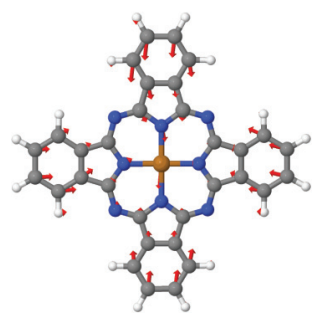

(c)

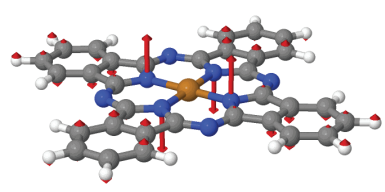

(e)

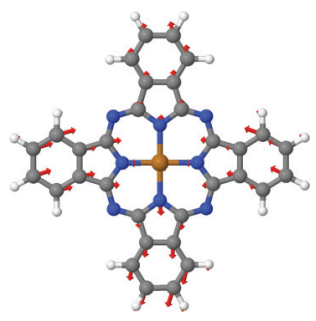

(b)

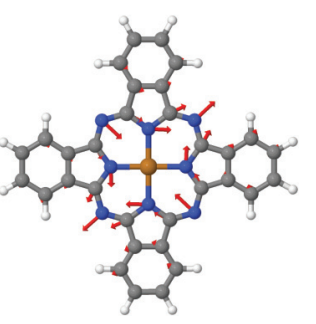

(d)

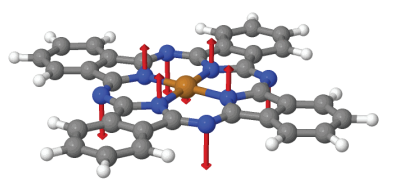

(f)
FIG. 19. (Color online) Selected vibrational modes of $\mathrm{CuPc}$ also observed in other $M \mathrm{Pc}$, and described as metal out-of-plane displacement (a), asymmetric (b), and symmetric (c) breathing, in-plane $\mathrm{N}-M-\mathrm{N}$ bending (d), out-of-plane asymmetric (e) and symmetric (f) $M-\mathrm{N}_{4}$ bending.
$M-\mathrm{N}_{4}$ stretching modes: this can be understood on the basis of their displacement vectors (Fig. 20), which indicate significant deformations of benzene rings and smaller displacements on atoms close to the metal. Vibrational modes which involve atomic displacements near the metal show larger shifts. For bendings the magnitude of the shifts can be tens of $\mathrm{cm}^{-1}$. There is a frequency increase from $\mathrm{Zn}$ to $\mathrm{Co}$, then a decrease for $\mathrm{Fe}$, and an increase again for $\mathrm{Cr}$. This is found for out-of-plane $M-\mathrm{N}_{4}$ bending, and for $M-\mathrm{N}_{2}$ stretching-bending modes.

In Table VI we compare our frequency values and the nature of vibrational modes of $\mathrm{CuPc}$ with those reported in Ref. 74

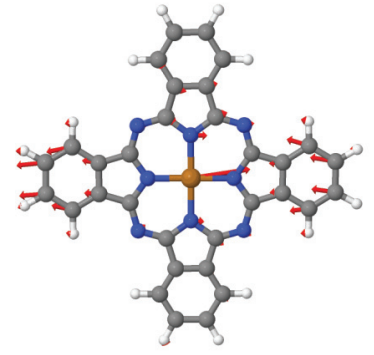

(a)

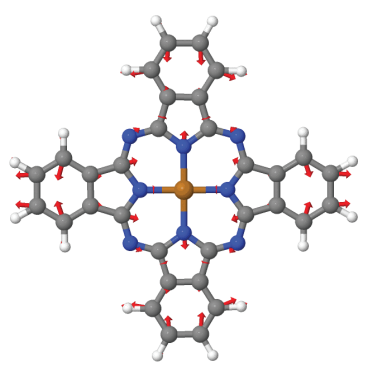

(c)

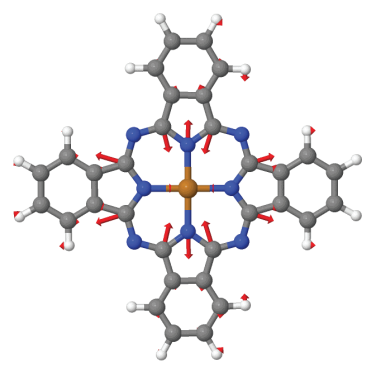

(e)

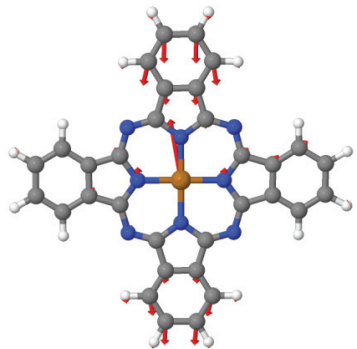

(b)

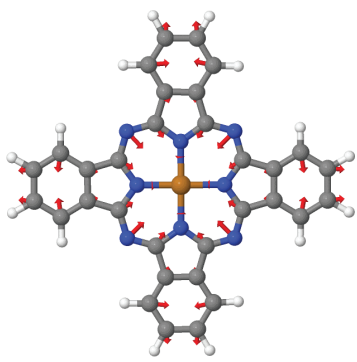

(d)

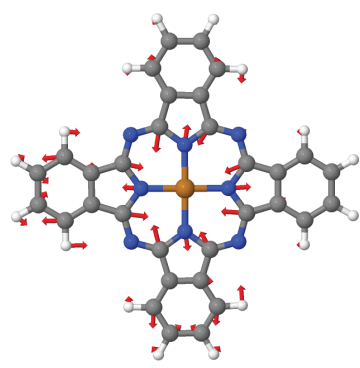

(f)
FIG. 20. (Color online) Selected vibrational modes of $\mathrm{CuPc}$, also observed in other $M$ Pc. $M-\mathrm{N}_{2}$ stretching-bending, (a) and (b); $M-\mathrm{N}_{4}$ asymmetric (c),(e) and symmetric (d),(f) stretching. 

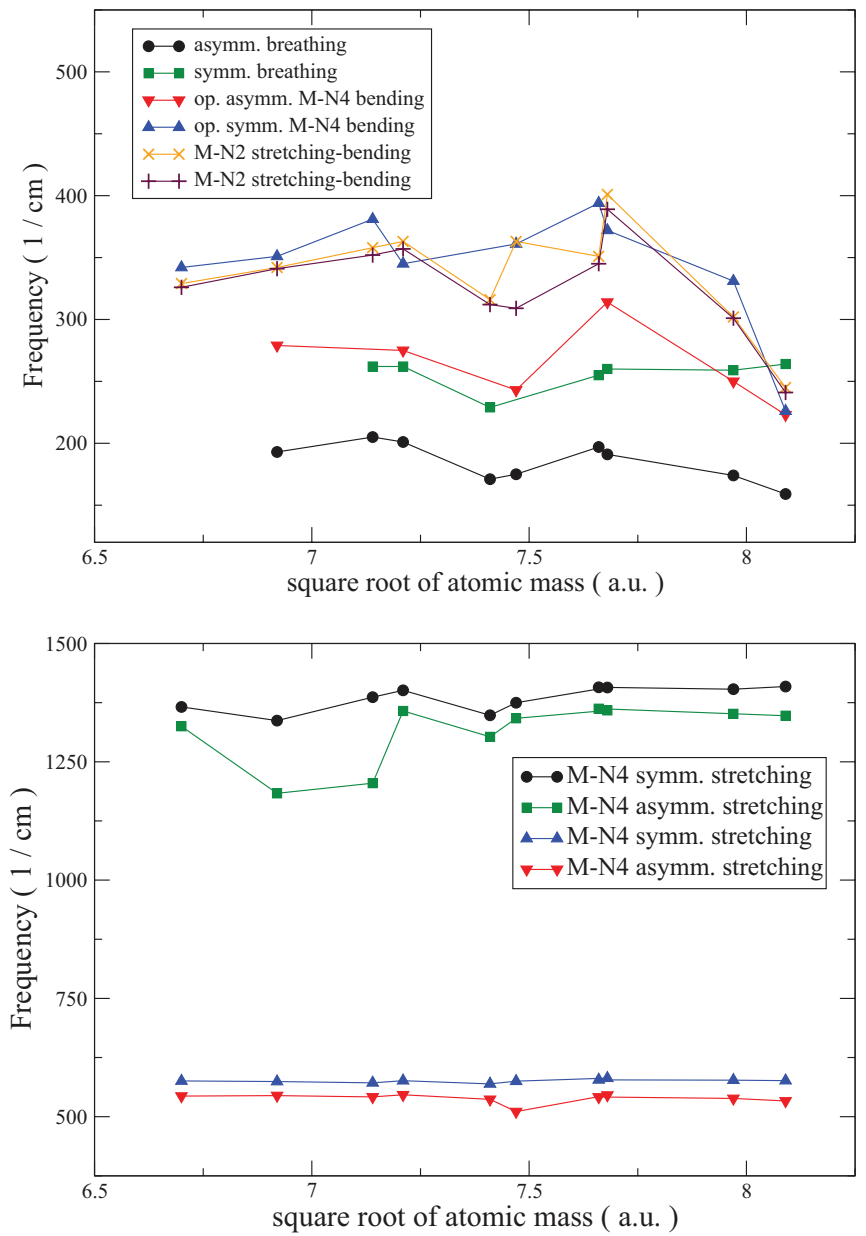

FIG. 21. (Color online) Frequencies of selected vibrational modes of $M$ Pc.

for the fluorinated system $\mathrm{FCuPc}$, particularly for those which were found to have a larger contribution to the energy-transfer process. Frequency values for $\mathrm{CuPc}$ become similar to results for $\mathrm{FCuPc}$ when the vibrational modes involve a small number of atoms, and when the atomic displacements are mainly from atoms closer to the metal. The above trends are also obtained with PBE.

\section{SUMMARY}

The magnetic and electronic properties of metal phthalocyanines $(M \mathrm{Pc})$ and fluorinated metal phthalocyanines $\left(\mathrm{F}_{16} \mathrm{MPc}\right)$ have been studied by spin density functional theory for the first row transition metals. $\mathrm{CaPc}$ and $\mathrm{AgPc}$ were also considered for comparison. A planar geometry with the metal lying inside the inner ring was obtained for all $M P c$ except for the cases $M=\mathrm{Ca}$ and $\mathrm{Sc}$, which are displaced from the plane

TABLE VI. Comparison of frequencies $\left(\mathrm{cm}^{-1}\right)$ of relevant vibrational modes of $\mathrm{CuPc}$ and $\mathrm{FCuPc}$.

\begin{tabular}{llrll}
\hline \hline $\mathrm{FCuPc}^{\mathrm{a}}$ & 175 & 983 & 1445 & 1570 \\
$\mathrm{CuPc}^{\mathrm{b}}$ & 201 & 1158 & 1401 & 1561 \\
\hline \hline
\end{tabular}

${ }^{\mathrm{a}}$ From Ref. 74

${ }^{\mathrm{b}}$ Our CA/DZP calculation. by 1.12 and $0.24 \AA$, respectively, in direct relation with their atomic sizes. The analysis of electronic states shows that the total magnetic moment is caused mainly by the electron density localized on the metal and its nearby atoms. Furthermore, when the hydrogen atoms are replaced by fluor atoms the magnetic moments do not change. The same behavior is found for the band-gap values. The $M-\mathrm{N}$ bond lengths of $\mathrm{F}_{16} M \mathrm{Pc}$ are slightly larger than the corresponding ones of $M \mathrm{Pc}$.

In the other hand, the analysis of the density of states (DOS) indicates a substantial change in the number, position, width, and height of the DOS peaks in $\mathrm{F}_{16} M \mathrm{Pc}$ in comparison to $M \mathrm{Pc}$. The fact that the total magnetic-moment value does not change can be explained by the charge density redistribution between the metal and its nitrogen and carbon neighbors. This rearrangement suggests a high flexibility of the electronic distribution which may account for the apparent independence of the $M$ Pc properties on the metal, also pointed out in previous works. ${ }^{75}$ However, DOS profiles obtained along the studied $d$-row series display different mixing between metal and ligand orbitals, giving rise to energy shifts of states.

The crystal-field approximation cannot account for the orbital mixing occurring in these compounds. Ligand orbitals $a_{1 u}$ and $e_{g}$ have the propensity to appear near the Fermi energy and they can become HOMO or LUMO when electronic repulsion shifts localized states on the metal or when electron pairing on the metal compensates repulsion between levels for both spins as in NiPc and ZnPc. Near the middle of the studied $d$ series, repulsion among unpaired electron densities causes different DFT functionals to disagree with respect to the electronic structure. However, we have found that in general, LDA results are valuable for the qualitative assessment of the metallic or ligand character HOMO and LUMO. Comparison with PBE results is useful to determine which metal localized orbitals are more strongly affected by the self-interaction error. The observed tendencies may be extrapolated to understand and predict the device performance of particular MPc employed in organic solar cells in which the exciton diffusion is affected by the energy ordering of orbitals. ${ }^{63}$

The band gap shows a steep drop from $\mathrm{CaPc}$ to $\mathrm{ScPc}$ when the $d$ shell is initiated. The largest value found in the $d^{2}-d^{4}$ interval is for $\mathrm{CrPc}$, and a second important decrease is predicted for MnPc. The semiconducting character is recovered for the $d^{7}-d^{10}$ series with low spin configuration. The crucial effect of the central metal in the electronic properties of $M \mathrm{Pc}$ helps to explain recent theoretical results about the energetics of their adsorption on an $\mathrm{Au}(111)$ surface, ${ }^{64}$ and the increasing impact of the $3 d$ states along the $\mathrm{ZnPc}-\mathrm{MnPc}$ series as determined by energy-loss and photoemission spectroscopy. ${ }^{76}$ Ongoing research regarding electron transport on these $M \mathrm{Pc}$ is being carried out in our group.

Based on the vibrational mode analysis, we find that, in general, frequencies increase when going from $\mathrm{Zn}$ to $\mathrm{Fe}$ if they correspond to asymmetric modes. There is an increment from either $\mathrm{Mn}$ or $\mathrm{Fe}$ to $\mathrm{Cr}$. The series $\mathrm{V}, \mathrm{Ti}$, and $\mathrm{Sc}$ shows a more irregular behavior. However, there is a consistent frequency decrease from $\mathrm{V}$ to $\mathrm{Sc}$ for vibrational modes which do not include breathing or metal out-of-plane movement. Vibrational frequencies values change a few tens of $\mathrm{cm}^{-1}$ when the metal in the central position is varied. This is an order of magnitude larger than the shifts provoked by molecular adsorption. ${ }^{77}$ 


\section{ACKNOWLEDGMENTS}

We thank P. Schwab for helpful discussions. We acknowledge financial support by the Deutsche Forschungsgemeinschaft (through TRR 80), PPPROALMEX-DAADConacyt binational support, Project SEP-CONACYT 152153,
CNS-IPICyT, Mexico, and TACC-Texas supercomputer center for providing computational resources. O.I.A.-F. thanks Camilo Garcia for valuable discussions and CONACyT for a postdoctoral fellowship. A.H.R. acknowledges support from the Marie-Curie Intra-European Fellowship program. *aromero@qro.cinvestav.mx

${ }^{1}$ J. Van den Brink and A. F. Morpurgo, Nature (London) 450, 117 (2007).

${ }^{2}$ N. Marom and L. Kronik, Appl. Phys. A 95, 159 (2009).

${ }^{3}$ S. A. V. Slyke, C. H. Chen, and C. W. Tang, Appl. Phys. Lett. 69, 2160 (1996).

${ }^{4}$ P. Peumans and S. R. Forrest, Appl. Phys. Lett. 79, 126 (2001).

${ }^{5}$ P. C. Kao, S. Y. Chu, Z. X. You, S. J. Liou, and C. A. Chuang, Thin Solid Films 498, 249 (2006).

${ }^{6}$ Z. N. Bao, A. J. Lovinger, and A. Dodabalapur, Appl. Phys. Lett. 69, 3066 (1996).

${ }^{7}$ R. W. I. de Boer, A. F. Stassen, M. F. Craciun, C. L. Mulder, A. Molinari, S. Rogge, and A. F. Morpurgo, Appl. Phys. Lett. 86, 262109 (2005).

${ }^{8}$ X. Chen and M. Alouani, Phys. Rev. B 82, 094443 (2010).

${ }^{9}$ H. Huang, W. Chen, and A. T. S. Wee, J. Phys. Chem. C 112, 14913 (2008).

${ }^{10}$ T. Wang, D. Ebeling, J. Yang, C. Du, L. F. Chi, H. Fuchs, and D. Yan, J. Phys. Chem. B 113, 2333 (2009).

${ }^{11}$ D. G. de Oteyza, E. Barrena, J. O. Osso, S. Sellner, and H. Dosch, J. Am. Chem. Soc. 128, 15002 (2006).

${ }^{12}$ D. G. de Oteyza, E. Barrena, Y. Zhang, T. N. Krauss, A. Turak, A. Vorobiev, and H. Dosch, J. Phys. Chem. C 113, 4234 (2009).

${ }^{13}$ L. Scudiero, K. W. Hipps, and D. E. Barlow, J. Phys. Chem. B 107, 2903 (2003).

${ }^{14}$ K. W. Hipps, L. Scudiero, D. E. Barlow, and M. P. Cooke, J. Am. Chem. Soc. 124, 2126 (2002).

${ }^{15}$ C. J. Drury, C. M. J. Mutsaers, C. M. Hart, M. Matters, and D. M. de Leeuw, Appl. Phys. Lett. 73, 108 (1998).

${ }^{16}$ Y. Watanable and K. Kudo, Appl. Phys. Lett. 87, 223505 (1995).

${ }^{17}$ R. Wisnieff, Nature (London) 394, 225 (1998).

${ }^{18}$ B. Crone, A. Dodabalapur, A. Gelperin, L. Torsi, H. E. Katz, A. J. Lovinger, and Z. Bao, Appl. Phys. Lett. 78, 2229 (2001).

${ }^{19}$ B. K. Crone, A. Dodabalapur, R. Sarpeshkar, A. Gelperin, H. E. Katz, and Z. Bao, J. Appl. Phys. 91, 10140 (2001).

${ }^{20}$ A. Dodabalapur, Z. Bao, A. Makhija, J. G. Laquindanum, V. R. Raju, Y. Feng, H. E. Katz, and J. Rogers, Appl. Phys. Lett. 73, 142 (1998).

${ }^{21}$ C. J. Brown, J. Chem. Soc. A 2488 (1968).

${ }^{22}$ J. F. Kirner, W. Dow, and W. R. Scheidt, Inorg. Chem. 15, 1658 (1976).

${ }^{23}$ W. R. Scheidt and W. Dow, J. Am. Chem. Soc. 99, 1101 (1977).

${ }^{24}$ F. Evangelista, V. Carravetta, G. Stefani, B. Jansik, M. Alagia, S. Stranges, and A. Ruocco, J. Chem. Phys. 126, 124709 (2007).

${ }^{25}$ Xiao and P. A. Dowben, J. Mater. Chem. 19, 2172 (2009).

${ }^{26}$ K. W. Hipps, X. Lu, X. D. Wang, and U. Mazur, J. Phys. Chem. 100, 11207 (1996).

${ }^{27}$ P. H. Lippel, R. J. Wilson, M. D. Miller, C. Woll, and S. Chiang, Phys. Rev. Lett. 62, 171 (1989).
${ }^{28}$ M. Filibian, P. Carretta, T. Miyake, Y. Taguchi, and Y. Iwasa, Phys. Rev. B 75, 085107 (2007).

${ }^{29}$ T. Schwieger, H. Peisert, M. S. Golden, M. Knupfer, and J. Fink, Phys. Rev. B 66, 155207 (2002).

${ }^{30}$ D. R. Tackley, G. Dent, and W. E. Smith, Phys. Chem. Chem. Phys. 2, 3949 (2000).

${ }^{31}$ K. A. Nguyen and R. Pachter, J. Chem. Phys. 114, 10757 (2001).

${ }^{32}$ N. Papageorgiou, Y. Ferro, E. Salomon, A. Allouche, J. M. Layet, L. Giovanneli, and G. LeLay, Phys. Rev. B 68, 235105 (2003).

${ }^{33}$ A. Rosa, G. Ricciardi, O. Gritsenko, and E. J. Baerends, Struct. Bonding (Berlin, Ger.) 112, 49 (2004).

${ }^{34}$ J. P. Gauyacq, F. D. Novaes, and N. Lorente, Phys. Rev. B 81, 165423 (2010)

${ }^{35}$ M. S. Liao and S. Scheiner, J. Chem. Phys. 114, 9780 (2001).

${ }^{36}$ L. Lozzi, S. Santucci, S. L. Rosa, B. Delley, and S. Picozzi, J. Chem. Phys. 121, 1883 (2004).

${ }^{37}$ B. Bialek, I. G. Kim, and J. I. Lee, Synth. Met. 129, 151 (2002).

${ }^{38}$ B. Bialek, I. G. Kim, and J. I. Lee, Thin Solid Films 436, 107 (2003).

${ }^{39}$ B. Bialek, I. G. Kim, and J. I. Lee, Surf. Sci. 526, 367 (2003).

${ }^{40}$ B. Bialek, I. G. Kim, and J. I. Lee, Thin Solid Films 513, 110 (2006).

${ }^{41}$ N. Marom, O. Hod, G. E. Scuseria, and L. Kronik, J. Chem. Phys. 128, 164107 (2008).

${ }^{42}$ S. H. Vosko, L. Wilk, and M. Nusair, Can. J. Phys. 58, 1200 (1980).

${ }^{43}$ A. D. Becke, Phys. Rev. A 38, 3098 (1988).

${ }^{44}$ J. P. Perdew, Phys. Rev. B 33, 8822 (1986).

${ }^{45}$ P. J. Stephens, F. J. Devlin, C. F. Chabalowski, and M. J. Frisch, J. Phys. Chem. 98, 11623 (1994).

${ }^{46}$ J. P. Perdew, K. Burke, and M. Ernzerhof, Phys. Rev. Lett. 77, 3865 (1996).

${ }^{47}$ J. P. Perdew, A. Ruzsinszky, J. Tao, V. N. Staroverov, G. E. Scuseria, and G. I. Csonka, J. Chem. Phys. 123, 062201 (2005).

${ }^{48}$ J. Heyd, G. E. Scuseria, and M. Ernzerhof, J. Chem. Phys. 118, 8207 (2003).

${ }^{49}$ S. Bhattacharjee, B. Brena, R. Banerjee, H. Wende, O. Eriksson, and B. Sanyal, Chem. Phys. 377, 96 (2010).

${ }^{50}$ J. M. Soler, E. Artacho, J. D. Gale, A. García, J. Junquera, P. Ordejón, and D. Sánchez-Portal, J. Phys.: Condens. Matter 14, 2745 (2002).

${ }^{51}$ J. L. Kahl, L. R. Faulkner, K. Dwarakanath, and H. Tachikawa, J. Am. Chem. Soc. 108, 5434 (1986).

${ }^{52}$ C. Clarisse, M. T. Riou, M. Gauneau, and M. Le Contellec, Electron. Lett. 24, 674 (1988).

${ }^{53}$ L. Li, Q. Tang, H. Li, W. Hu, X. Yang, Z. Shuai, Y. Liu, and D. Zhu, Pure Appl. Chem. 80, 2231 (2008).

${ }^{54}$ H.-Z. Chen, C. Pan, and M. Wang, Nanostruct. Mater. 11, 523 (1999). 
${ }^{55}$ H. Tada, H. Touda, M. Takada, and K. Matsushige, Appl. Phys. Lett. 76, 873 (2000).

${ }^{56}$ D. E. Barlow and K. W. Hipps, J. Phys. Chem. B 104, 5993 (2000).

${ }^{57}$ Y. L. Pan, Y. J. Wu, L. B. Chen, Y. Y. Zhao, Y. H. Shen, F. M. Li, S. Y. Shen, and D. H. Huang, Appl. Phys. A 66, 569 (1998).

${ }^{58}$ N. Marom and L. Kronik, Appl. Phys. A 95, 165 (2009).

${ }^{59}$ G. A. Williams, B. N. Figgis, R. Mason, S. A. Mason, and P. E. Fielding, J. Chem. Soc. Dalton Trans. 1688 (1980).

${ }^{60}$ J. M. Robertson and I. Woodward, J. Chem. Soc. 219 (1937).

${ }^{61}$ C. Ruan, V. Mastryukov, and M. Fink, J. Chem. Phys. 111, 3035 (1999).

${ }^{62}$ X. Shen, L. Sun, Z. Yi, E. Benassi, R. Zhang, Z. Shen, S. Sanvito, and S. Hou, Phys. Chem. Chem. Phys. 12, 10805 (2010).

${ }^{63}$ I. Bruder, J. Schöneboom, R. Dinnebier, A. Ojala, S. Schäfer, R. Sens, P. Erk, and J. Weis, Org. Electron. 11, 377 (2010).

${ }^{64}$ Y. Y. Zhang, S. X. Du, and H. J. Gao, Phys. Rev. B 84, 125446 (2011).

${ }^{65}$ The broadening scheme is meant to mimic the effect of interactions with the environment only in an approximate way; this also helps to address bands from orbitals of the isolated molecule. However, the location of the Fermi level within the HOMO band must be taken carefully, mainly in configurations considered as metallic.

${ }^{66}$ R. S. Mulliken, J. Chem. Phys. 36, 3428 (1962).
${ }^{67}$ D. Stradi, C. Díaz, F. Martín, and M. Alcamí, Theor. Chem. Acc. 128, 497 (2011).

${ }^{68}$ B. Brena, C. Puglia, M. de Simone, M. Coreno, K. Tarafder, V. Feyer, R. Banerjee, E. Göthelid, B. Sanyal, P. M. Oppeneer, and O. Eriksson, J. Chem. Phys. 134, 074312 (2012).

${ }^{69}$ K. Nakamura, Y. Kitaoka, T. Akiyama, T. Ito, M. Weinert, and A. J. Freeman, Phys. Rev. B 85, 235129 (2012).

${ }^{70}$ A. Mugarza, R. Robles, C. Krull, R. Korytár, N. Lorente, and P. Gambardella, Phys. Rev. B 85, 155437 (2012).

${ }^{71}$ F. Roth, A. König, R. Kraus, M. Grobosch, T. Kroll, and M. Knupfer, Eur. Phys. J. B 74, 339 (2010).

${ }^{72}$ F. Petraki, H. Peisert, U. Aygül, F. Latteyer, J. Uihlein, A. Vollmer, and T. Chassé, J. Phys. Chem. C 116, 11110 (2012).

${ }^{73}$ E. Minamitani, D. Matsunaka, N. Tsukahara, N. Takagi, M. Kawai, and Y. Kim, e-J. Surf. Sci. Nanotechnol. 10, 38 (2012).

${ }^{74}$ F.-C. Wu, H.-L. Cheng, C.-H. Yen, J.-W. Lin, S.-J. Liu, W.-Y. Chou, and F.-C. Tang, Phys. Chem. Chem. Phys. 12, 2098 (2010).

${ }^{75}$ A. F. Takács, F. Witt, S. Schmaus, T. Balashov, M. Bowen, E. Beaurepaire, and W. Wulfhekel, Phys. Rev. B 78, 233404 (2008).

${ }^{76}$ M. Grobosch, C. Schmidt, R. Kraus, and M. Knupfer, Org. Electron. 11, 1483 (2010).

${ }^{77}$ G. S. S. Saini, S. D. Dogra, K. Sharma, S. Singh, S. K. Tripathi, V. Sathe, and R. K. Singh, Vib. Spectrosc. 57, 61 (2011). 